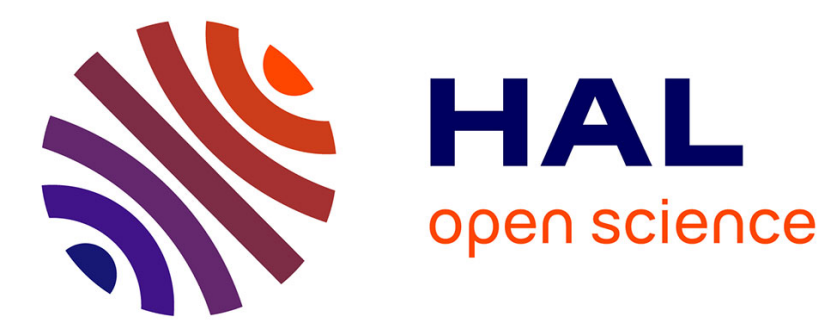

\title{
Universal social welfare orderings and risk
}

Marc Fleurbaey, Stéphane Zuber

\section{To cite this version:}

Marc Fleurbaey, Stéphane Zuber. Universal social welfare orderings and risk. 2021. halshs-03289160

\section{HAL Id: halshs-03289160 \\ https://shs.hal.science/halshs-03289160}

Submitted on 16 Jul 2021

HAL is a multi-disciplinary open access archive for the deposit and dissemination of scientific research documents, whether they are published or not. The documents may come from teaching and research institutions in France or abroad, or from public or private research centers.
L'archive ouverte pluridisciplinaire HAL, est destinée au dépôt et à la diffusion de documents scientifiques de niveau recherche, publiés ou non, émanant des établissements d'enseignement et de recherche français ou étrangers, des laboratoires publics ou privés. 


\section{Documents de Travail du

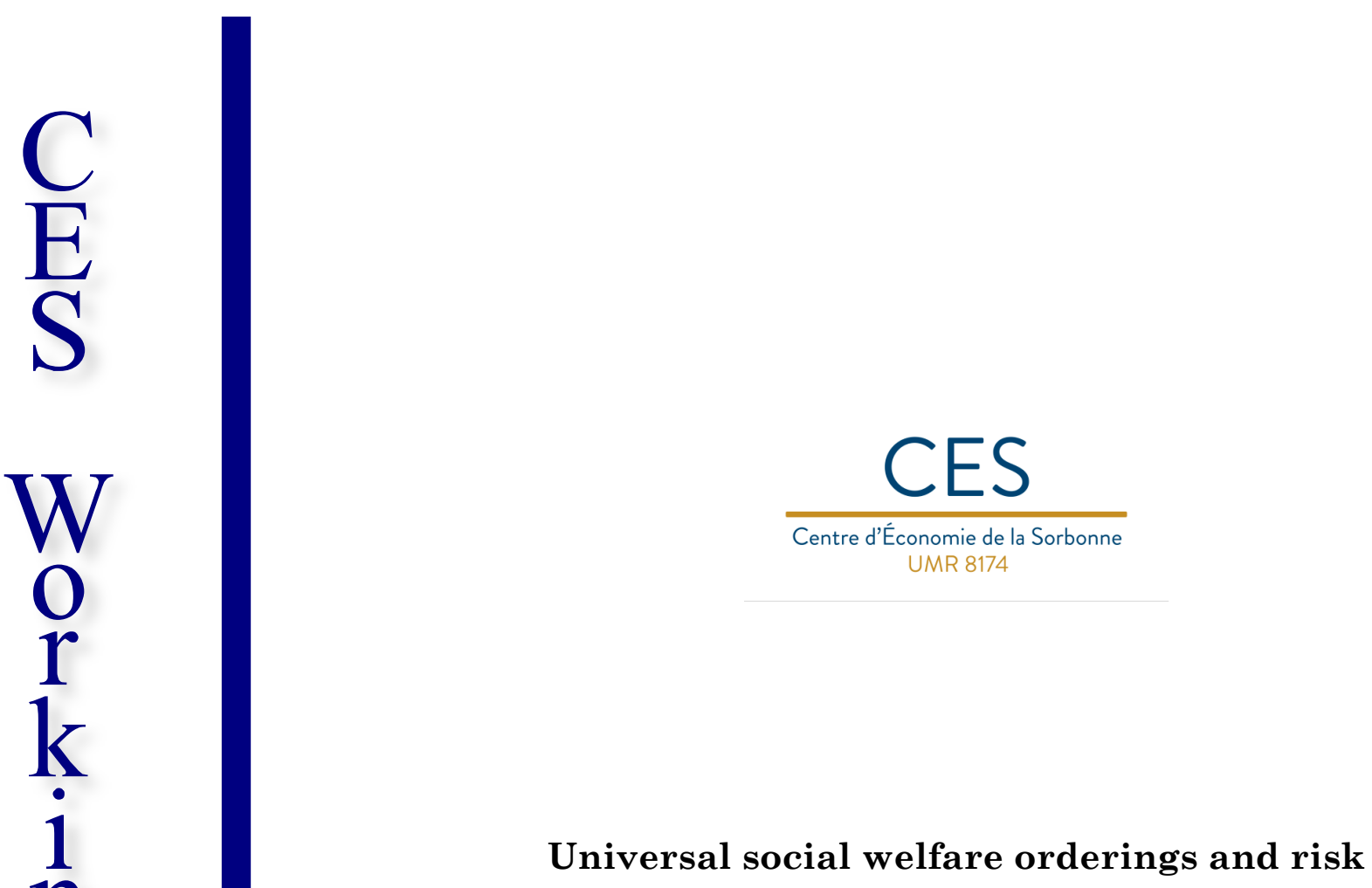

Marc Fleurbaey, Stéphane ZuBER

2021.18

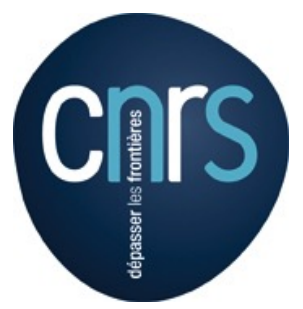




\title{
Universal social welfare orderings and risk*
}

\author{
Marc Fleurbaey $^{a} \quad$ Stéphane Zuber ${ }^{b}$
}

June 2021

\begin{abstract}
How to evaluate and compare social prospects when there may be a risk on i) the actual allocation people will receive; ii) the existence of these future people; and iii) their preferences? This paper investigate this question that may arise when considering policies that endogenously affect future people, for instance climate policy. We show that there is no social ordering that meets minimal requirements of fairness, social rationality, and respect for people's ex ante preferences. We explore three ways to avoid this impossibility. First, if we drop the ex ante Pareto requirement, we can obtain fair ex post criteria that take an (arbitrary) expected utility of an equally-distributed equivalent level of well-being. Second, if the social ordering is not an expected utility, we can obtain fair ex ante criteria that assess uncertain individual prospects with a certainty-equivalent measure of well-being. Third, if we accept that interpersonal comparisons rely on VNM utility functions even in absence of risk, we can construct expected utility social orderings that satisfy of some version of Pareto ex ante.
\end{abstract}

Keywords: Fairness, social risk, intergenerational equity.

JEL Classification numbers: D63, D81.

* This research has been supported by the Agence nationale de la recherche through the FairClimPop project (ANR-16-CE03-0001-01) and the Investissements d'Avenir program (ANR-17EURE-01).

${ }^{a}$ Paris School of Economics - CNRS, France. Address: 48, Boulevard Jourdan, 75014 Paris. E-mail: Marc.Fleurba@psemail.eu.

${ }^{b}$ Paris School of Economics - CNRS, France. Address: Centre d'Économie de la Sorbonne, 106-112 boulevard de l'Hôpital, 75013 Paris, France. E-mail: Stephane.Zuber@univ-paris1.fr. 


\section{Introduction}

To unify evaluations and comparisons of living standards for individuals and populations of possibly different sizes and preferences, Fleurbaey and Tadenuma (2014) have introduced the concept of universal social orderings. Such universal social orderings are defined over pairs of allocation and preference profiles that may differ in size, e.g., making it possible to compare the situation of the European Union in 1999 with that of India in 2015. The aim of this paper is to extend this theory to risk. Indeed, many relevant economic situations involve risk (or uncertainty) about i) the actual allocation people will receive; ii) their preferences; and iii) their existence and the size of the population.

This is illustrated by the question of climate change and the design of climate policies. It is widely recognized that there are deep uncertainties about how climate change may affect the economy (Weitzman, 2009; IPCC, 2014). But it is also widely recognized that the size of the future population is also very uncertain, and this raises crucial questions, including ethical ones (Millner, 2013; Scovronick et al., 2017). A few papers have also highlighted the issue of uncertainty about future preferences and its consequences for climate policy (Beltratti, Chichilnisky and Heal, 1998; Ayong Le Kama, 2001; Ayong Le Kama and Schubert, 2004). But they do not discuss in details the welfare framework to be used for evaluating such changes. It is noteworthy that, in the workhorse welfare framework for analyzing climate policies, namely the expected discounted utility (EDU) model, there is a confusion between risk aversion, inequality aversion and the intertemporal elasticity of substitution, which makes it unclear how individual wellbeing should be evaluated (Atkinson et al., 2009; Dasgupta, 2008; Anthoff, Tol and Yohe, 2009).

Uncertainty about future preferences, and therefore preference diversity, is a real challenge when analyzing the impact of public policies because it raises the problem of wellbeing measurement and comparison for individuals having noncomparable utilities. This has been one of the central issues of social choice theory and welfare economics. For the case in which individuals face risk, the seminal contribution is Harsanyi's (1955) aggregation theorem. This theorem, which in- 
volves the Pareto principle applied in risky situations, characterizes utilitarianism as a sum of Von Neumann-Morgenstern (henceforth VNM) individual utilities. Hence, Harsanyi recommended to rely on these specific functions for wellbeing measurement and comparisons. ${ }^{1}$ This assumption was made in his initial paper and in most of the subsequent literature (Broome, 1991; Diamond, 1967; Epstein and Segal, 1992). But this remains a controversial assumption (as argued for instance by Chambers and Echenique, 2012, and Fleurbaey and Zuber, 2017) and many alternative welfare measures have been proposed in the literature. In particular, it is debatable whether people's risk attitudes should play a role in interpersonal comparisons made in absence of risk. In this paper, we consider alternative measures that are based only on ordinal preferences and the allocation people receive, and are independent of risk attitudes in contexts that are devoid of risk.

The aim of the present paper is to introduce a framework that can be used to explicitly address the issue of welfare measurement in situations involving preference diversity, preference uncertainty, and uncertainty about the identity of future people. Following the fair social choice approach (Fleurbaey and Maniquet, 2011), wellbeing measures are preference-based and embody fairness principles expressed in terms of the resources (income, environmental goods, health) available to individuals. Such measures allow for preference diversity within and across generations but traditionally, they do not take account of risk attitudes for comparisons in riskless contexts. This paper is part of a more general project to extend and bridge two separate branches the literature: the theory of fair social orderings and the theory of social choice under risk.

The following sections are organized as follows. Section 2 contains the presentation of the framework and main definitions. Section 3 offers an introduction to fair social orderings and proves the impossibility of extending it to risk without compromising basic principles of fairness, rationality, or respect for preferences.

\footnotetext{
${ }^{1}$ There is an issue on how these VNM utilities should be normalized. The most widespread proposal is to scale the utility functions by setting the utility of a "good" option (generally the best) equal to one and the utility of a "bad" option (generally the worst) equal to zero. This has been called "relative utilitarianism" by Dhillon and Mertens (1999).
} 
Section 4 focuses on the tension between rationality and respect for preferences, and is devoted to the characterization of the ex ante and the ex post approaches to social welfare measurement. Section 5 revisits the well-being measures coming from the theory of fair social orderings and shows that allowing risk attitudes to matter even in absence of risk, for interpersonal comparisons, opens additional possibilities for reconciling social rationality and the Pareto principle.

\section{Framework}

\subsection{The framework}

The set of potential individuals who may or may not exist is $\mathbb{N}^{2}$ In any social alternative, only a subpopulation $N \in \mathcal{N}$ exists, where $\mathcal{N}$ is the set of non-empty finite subsets of $\mathbb{N}$.

A social alternative $a$ specifies the population $N$ that exists and the distribution of preferences and goods $a=\left(x_{N}, R_{N}\right)=\left(\left(x_{i}, R_{i}\right)_{i \in N}\right)$, where each $x_{i}$ is a bundle in $\mathcal{X}=\mathbb{R}_{+}^{k}$ (with $k \in \mathbb{Z}_{++}$) and each $R_{i}$ is a preference ordering in a set $\mathcal{R}$ that we describe below. We denote the set of social alternatives $\mathcal{A}=\bigcup_{N \in \mathcal{N}}(\mathcal{X} \times \mathcal{R})^{N}$. For any $a \in \mathcal{A}, N(a)$ is the set of individuals who exist in alternative $a$ and $n(a)=|N(a)|$ is the number of individuals in the alternative. For two disjoint populations $N$ and $M,\left(\left(x_{N}, R_{N}\right),\left(x_{M}, R_{M}\right)\right)$ denotes the alternative $\left(x_{N \cup M}, R_{N \cup M}\right)$.

We assume that it is not always known for sure what the final allocation of consumption will be, nor what set of individuals will eventually exist. To model this, we assume that there exists an infinite set of states of the world $\mathcal{S}$, with typical element $s \in \mathcal{S}$. We denote $\mathcal{E}$ a $\sigma$-algebra over $\mathcal{S}$, so that $(\mathcal{S}, \mathcal{E})$ is a measurable space. There is a probability measure $P$ on the measurable space $(\mathcal{S}, \mathcal{E})$, which is supposed to be objective (or it is the belief used in the

\footnotetext{
${ }^{2}$ Notation: $\mathbb{N}$ denotes the set of positive integers; $\mathbb{R}$ (resp. $\mathbb{R}_{+}, \mathbb{R}_{++}$) the set of real numbers (resp. non-negative, positive real numbers). For a set $D$ and any $n \in \mathbb{N}, D^{n}$ is the $n$-fold Cartesian product of $D$. Also, for two sets $D$ and $E, D^{E}$ denotes the set of mappings from $E$ into $D$.
} 
evaluation). ${ }^{3}$

A social prospect $f$ is a finitely-valued function from $\mathcal{S}$ to $\mathcal{A}$, which is supposed to be $\mathcal{E}$-measurable. For $s \in \mathcal{S}, f(s) \in \mathcal{A}$ is therefore the alternative induced by the prospect $f$ in state $s$. We denote by $\mathcal{F}$ the set of all such social prospects. For any random variable $K$, i.e., any $\mathcal{E}$-measurable function $K: \mathcal{S} \rightarrow \mathbb{R}$, its expected value is $\mathbb{E}[K]=\int_{\mathcal{S}} K(s) d P(s)$.

For a prospect $f \in \mathcal{F}$ and a state of the world $s \in \mathcal{S}$, whenever $i \in N(f(s))$, $x_{i}(f(s))$ denotes the bundle allocated to individual $i$ in state of the world $s \in \mathcal{S}$ and $R_{i}(f(s))$ the preference ordering of individual $i$ in that state. For any $i \in \mathbb{N}$ and any $R \in \mathcal{R}$, we let

$$
E_{i, R}(f)=\left\{s \in \mathcal{S} \mid i \in N(f(s)) \text { and } R_{i}(f(s))=R\right\}
$$

be the event in which individual $i$ exists with preferences $R$. We let $P_{i, R}(f)=$ $P\left(E_{i, R}(f)\right)$ denote the probability of this event. Similarly, for a subpopulation $N \in \mathcal{N}$ and a profile of preferences $R_{N}=\left(R_{i}\right)_{i \in N} \in \mathcal{R}^{N}$, we define

$$
E_{N, R_{N}}(f)=\left\{s \in \mathcal{S} \mid N(f(s))=N \text { and } R_{i}(f(s))=R_{i} \text { for all } i \in N\right\}
$$

and we let $P_{N, R_{N}}(f)=P\left(E_{N, R_{N}}(f)\right)$ be the probability that a specific subpopulation with specific preferences exists.

By convention, for any $a \in \mathcal{A}$, we let $a$ denote the prospect $f \in \mathcal{F}$ such that $f(s)=a$ for all $s \in \mathcal{S}$. These are sure prospects yielding the same alternative in all states of the world. Similarly, let $\mathcal{A} \subset \mathcal{F}$ also denote the set of sure prospects.

It remains to specify how individuals rank different prospects and alternatives. To do so, let us first focus on the case in which an individual exists for sure with given preferences. We let $\mathcal{L}$ denote the set of individual (consumption) prospects that are $\mathcal{E}$-measurable finitely-valued functions $\ell$ from $\mathcal{S}$ to $\mathcal{X}$. Individual preferences for individual $i$ are represented by a complete and transitive

\footnotetext{
${ }^{3}$ It is well known that heterogenous beliefs create problems for social choice, and in this paper we want to focus on more fundamental problems which occur even in absence of belief heterogeneity.
} 
relation $R_{i}$ over $\mathcal{L}$ (with associated indifference relation $I_{i}$ and strict preference $\left.P_{i}\right)$. More specifically, an individual preference ordering $R_{i}$ belongs to $\mathcal{R}$ if there exists an increasing and continuous Von Neumann-Morgenstern (WNM) function $v_{i}: \mathcal{X} \rightarrow \mathbb{R}$, which is unique up to an increasing affine transformation and such that, for all $\ell, \ell^{\prime} \in \mathcal{L}$ :

$$
\ell R_{i} \ell^{\prime} \Longleftrightarrow \mathbb{E}\left[v_{i} \circ \ell\right] \geq \mathbb{E}\left[v_{i} \circ \ell^{\prime}\right]
$$

In the sequel, we adopt the convention that $v_{R_{i}}$ denotes an arbitrarily chosen VNM function associated with the preference ordering $R_{i}$.

These preferences induce preferences on sure bundles in $\mathcal{X}$. Indeed, for any $x, y \in \mathcal{X}$, we say that $x$ is weakly preferred to $y(x R y)$ if there exist $\ell, \ell^{\prime} \in \mathcal{L}$ such that $\ell(s)=x$ and $\ell^{\prime}(s)=y$ for all $s \in \mathcal{S}$, and $\ell R \ell^{\prime}$. Preferences restricted to sure prospects are therefore complete and representable by a continuous utility function. Our assumptions about $\mathcal{R}$ also entail that individuals are expected utility maximizers. Experimental economics have highlighted that expected utility may not be behaviorally realistic, as individuals often violate the VNM-independence property and do not necessarily have well-defined beliefs. However, the assumption remains normatively relevant to represent the interests of individuals.

One difficulty we encounter is that, for a given social prospect, an individual $i \in \mathbb{N}$ may not exist in all states of the world and may have different preferences in different states. We do not assume that individuals can compare two prospects where they do not exist with the same probability or do exist but with different preferences. Instead, we rely on conditional expected utilities to represent individual interests for subsets of states with fixed preferences. For any $E \in \mathcal{E}$, let $\mathcal{E}_{E}=\left\{E^{\prime} \cap E \mid E^{\prime} \in \mathcal{E}\right\}$, which is a sigma-algebra over $E .{ }^{4}$ We define the conditional probability $P_{E}: \mathcal{E}_{E} \rightarrow[0,1]$ by $P_{E}\left(E^{\prime}\right)=P\left(E^{\prime}\right) / P(E)$, and $\left(E, \mathcal{E}_{E}, P_{E}\right)$ is a probability space. For any $\mathcal{E}_{E}$-measurable function $K: E \rightarrow \mathbb{R}$, its conditional expected value is $\mathbb{E}_{E}[K]=\int_{E} K(s) d P_{E}(s)$. For a social prospect $f$ where individual $i$ exists in some event $E$ with preferences $R_{i}, \mathbb{E}_{E}\left[v_{R_{i}} \circ x_{i}(f)\right]$ is the

\footnotetext{
${ }^{4}$ Remark that $\mathcal{E}_{E}$ is well-defined given that a sigma-algebra is closed by intersection.
} 
conditional expected utility of this individual. We can use this expression to represent individual preferences conditional on existence with given preferences in some states of the world.

Regarding the social ranking of prospects, we also make the following assumption.

A social welfare ordering $\succsim$ is a complete and transitive relation over $\mathcal{F}$. Furthermore, for all $N \in \mathcal{N}, R_{N}, R_{N}^{\prime} \in \mathcal{R}^{N}$ and $x_{N} \in \mathcal{X}^{N}$, the sets $\left\{x_{N}^{\prime} \in \mathcal{X}^{N} \mid\left(x_{N}^{\prime}, R_{N}^{\prime}\right) \succsim\left(x_{N}, R_{N}\right)\right\}$ and $\left\{x_{N}^{\prime} \in \mathcal{X}^{N} \mid\left(x_{N}, R_{N}\right) \succsim\right.$ $\left.\left(x_{N}^{\prime}, R_{N}^{\prime}\right)\right\}$ are closed.

The second part of the definition of the social welfare ordering is a continuity property. Note that, at this stage, we do not make any expected utility assumption for the social ranking.

\subsection{Well-being measures}

As discussed in the introduction, a key question when comparing different populations is to determine how allocations given to people with different preferences may be compared. This question of well-being measurement and inter-personal comparisons of individual situations has been a central issue in social choice and welfare economics. Following Pareto, Samuelson and Arrow, economists have often argued that we should only use ordinal non-comparable preferences. But of course, as argued in Karni (2003), we do need to compare individual situations to formulate meaningful distributive judgements. In this paper, and following the literature on fair allocation of resources, we propose to compare upper-contour sets. We will define index numbers for interpersonal comparisons that will correspond to some valuation of upper-contour sets.

For any $x \in \mathcal{X}$ and $R \in \mathcal{R}$, we denote $U(x, R)=\left\{x^{\prime} \in \mathcal{X} \mid x^{\prime} R x\right\}$ the (closed) upper-contour set of $x$ according to $R .^{5}$ Denote $\mathcal{U}=\cup_{(x, R) \in \mathcal{X} \times \mathcal{R}} U(x, R)$ the set of all upper-contour sets, with typical element $U$. Assigning numbers to uppercontour sets is done by what we will call set functions, i.e., functions $\psi: \mathcal{U} \rightarrow \mathbb{R}_{+}$

\footnotetext{
${ }^{5}$ We use the standard notation $P$ to denote strict preference and $I$ to denote indifference.
} 
such that $\psi(\mathcal{X})=0$ and $\psi(\mathcal{U})=\mathbb{R}_{+} \cdot{ }^{6}$ We say that an upper-contour set $U$ is strictly above another upper-contour set $U^{\prime}$ if, for all $x \in U$ there exists $x^{\prime} \in U^{\prime}$ such that $x \gg x^{\prime}$. A set function is increasing if for all $U, U^{\prime} \in \mathcal{U}$ such that $U$ is strictly above $U^{\prime}$, one has $\psi(U)>\psi\left(U^{\prime}\right)$.

A possible measurement of individual well-being consists in fixing a set function $\psi$ and defining the utility of individual $i$ with preferences $R_{i}$ and allocation $x_{i}$ as $u_{\psi}\left(x_{i}, R_{i}\right)=\psi\left(U\left(x_{i}, R_{i}\right)\right)$. Notice that, if $\psi$ is increasing, $u_{\psi}\left(., R_{i}\right)$ is a proper representation of preferences in the sense that, for all sure individual prospects $x, x^{\prime} \in \mathcal{X}, x R_{i} x^{\prime} \Longleftrightarrow u_{\psi}\left(x, R_{i}\right) \geq u_{\psi}\left(x^{\prime}, R_{i}\right)$.

Well-being measurement using set functions may appear quite abstract. Let us therefore mention two methods for interpersonal comparisons that have been widely discussed in the literature and are special cases of this approach (they are illustrated in the case of two goods in Fig. 1):

1. The money-metric approach (Samuelson and Swamy, 1974). Let $p \in \mathbb{R}_{++}^{k}$ be a reference price vector. In the money-metric approach, well-being is measured by the minimal expenditure needed at price $p$ to reach a level of satisfaction equivalent to $x$ :

$$
e_{p}(x, R)=\min \{p z \mid z \in U(x, R)\}
$$

With the set function $\psi_{p}(U)=\min \{p z \mid z \in U\}$, one obtains $e_{p}(x, R)=$ $u_{\psi_{p}}(x, R)$.

2. The Pazner-Schmeidler equivalence approach (Pazner and Schmeidler, 1978). Let $\omega \in \mathcal{X} \backslash\{0\}$ be a reference bundle. In the Pazner-Schmeidler equivalence approach, well-being is measured by the fraction of $\omega$ which is considered as good as $x$ :

$$
e_{\omega}(x, R)=\min \left\{e \in \mathbb{R}_{+} \mid e \omega \in U(x, R)\right\}
$$

\footnotetext{
${ }^{6} \mathrm{We}$ choose to normalize set functions without loss of generality. $\psi(\mathcal{X})$ is the value of the upper contour set at bundle giving 0 of each good. $\psi(\mathcal{U})=\mathbb{R}_{+}$means that the co-domain of $\psi$ is the entire set of non-negative real numbers.
} 


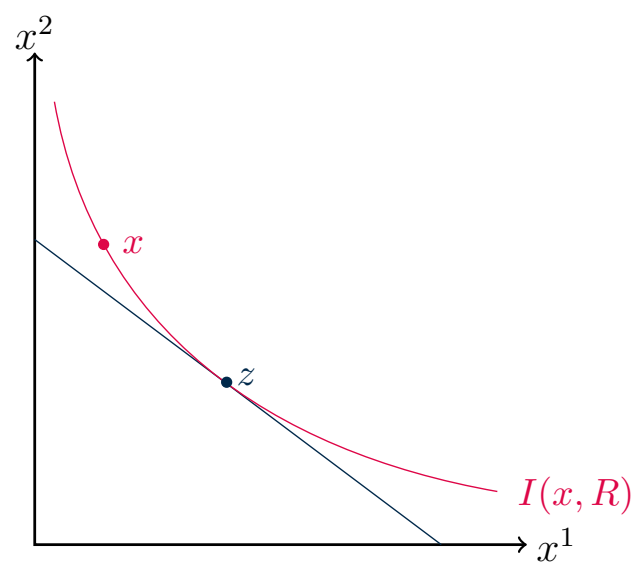

(a) Money metric

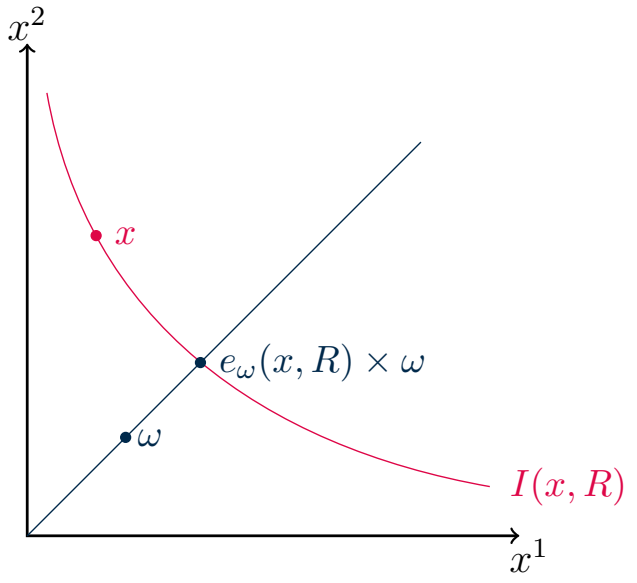

(b) Pazner-Schmeidler equivalence

Figure 1: Examples of well-being measures using ordinal information

Note: The set $I(x, R)$ is the set of bundles that are indifferent to $x$ according to the preference ordering $R$. In the left panel, the allocation $z$ is the one minimizing the budget $p z$ to reach a level of satisfaction equivalent to $\mathrm{x}$. In the right panel, the allocation $\omega$ is the reference allocation and $e_{\omega}(x, R) \omega$ is the allocation equivalent to $x$ when preferences are $R$.

With the set function $\psi_{\omega}(U)=\min \{e \mid e \omega \in U\}$, one obtains $e_{\omega}(x, R)=$ $u_{\psi_{\omega}}(x, R)$.

Well-being functions based on set functions are measures of individual wellbeing when there is no risk. Under risk, there also exist ex-ante measures of individual well-being. The certainty equivalent is one such measure. Usually, the certainty equivalent is defined for income or for consumption of a single good, but here we deal with bundles and thus need to adapt the definition. Denote $1_{k}$ the vector $(1, \cdots, 1) \in \mathcal{X}$, and for any $e \in \mathbb{R}_{+} e \cdot 1_{k}=(e, \cdots, e) \in \mathcal{X}$. For any individual $i$, any preference $R_{i}$, any $f \in \mathcal{F}$ such that $E_{i, R_{i}}(f) \neq \emptyset$, the certaintyequivalent proportional consumption bundle for $f$ when the individual exists with preferences $R_{i}$, denoted $c e_{i, R_{i}}(f)$, is the vector $e \cdot 1_{k} \in \mathcal{X}$ such that

$$
v_{R_{i}}\left(e \cdot 1_{k}\right)=\mathbb{E}_{E_{i, R_{i}}(f)}\left[v_{R_{i}}\left(x_{i}(f)\right)\right]
$$

Note that our notion of certainty-equivalent is compatible with cases in which individuals do not exist for sure in all states of the world, and may have different 
preferences in different events. Simply, there is a different certainty-equivalent for the events $E_{i, R}(f)$ associated with different preferences $R$ for the individual.

\section{Social welfare measurement and risk: general results}

\subsection{Fair universal social welfare orderings without risk}

Let us introduce some principles for the social evaluation of allocations. We will consider that these principles define basic requirements for individual and social welfare measurement. The first principle is a version of the Pareto principle when there is no risk and when there is only one individual. It states that whenever an individual is alone in society and prefers an allocation, this allocation is socially better.

Pareto for individual allocation. For all $i \in \mathbb{N}$, all $R_{i} \in \mathcal{R}$, and all $x_{i}, x_{i}^{\prime} \in \mathcal{X}$, $\left(x_{i}, R_{i}\right) \succsim\left(x_{i}^{\prime}, R_{i}\right)$ if and only if $x_{i} R_{i} x_{i}^{\prime}$.

Pareto for individual allocation is based on the idea of respecting preferences. The next axiom, Preference monotonicity, is based on the same idea but extends it to the case in which preferences change. Suppose that, after some change in preferences, a bundle unambiguously moves up in the individual ranking in the sense that it is preferred to more bundles. In that case, we may want to declare the individual consuming this bundle better off from the change in preferences.

Preference monotonicity. For all $i \in \mathbb{N}$, all $R_{i}, R_{i}^{\prime} \in \mathcal{R}$, and all $x_{i} \in \mathcal{X}$, if

$$
U\left(x_{i}, R_{i}\right) \subsetneq U\left(x_{i}, R_{i}^{\prime}\right) \text { then }\left(x_{i}, R_{i}\right) \succsim\left(x_{i}, R_{i}^{\prime}\right) .
$$

Preference monotonicity is related to the following property of informational parsimony: individual well-being comparisons only depend on ordinal preferences at the concerned allocations (i.e., only on the indifference curves). This is reminiscent of Hansson Independence, a weakening of Arrow Independence, and allows possibility theorems in social choice theory without assuming that preferences are comparable (see Hansson, 1973; Fleurbaey, Suzumura and Tadenuma, 2005). 


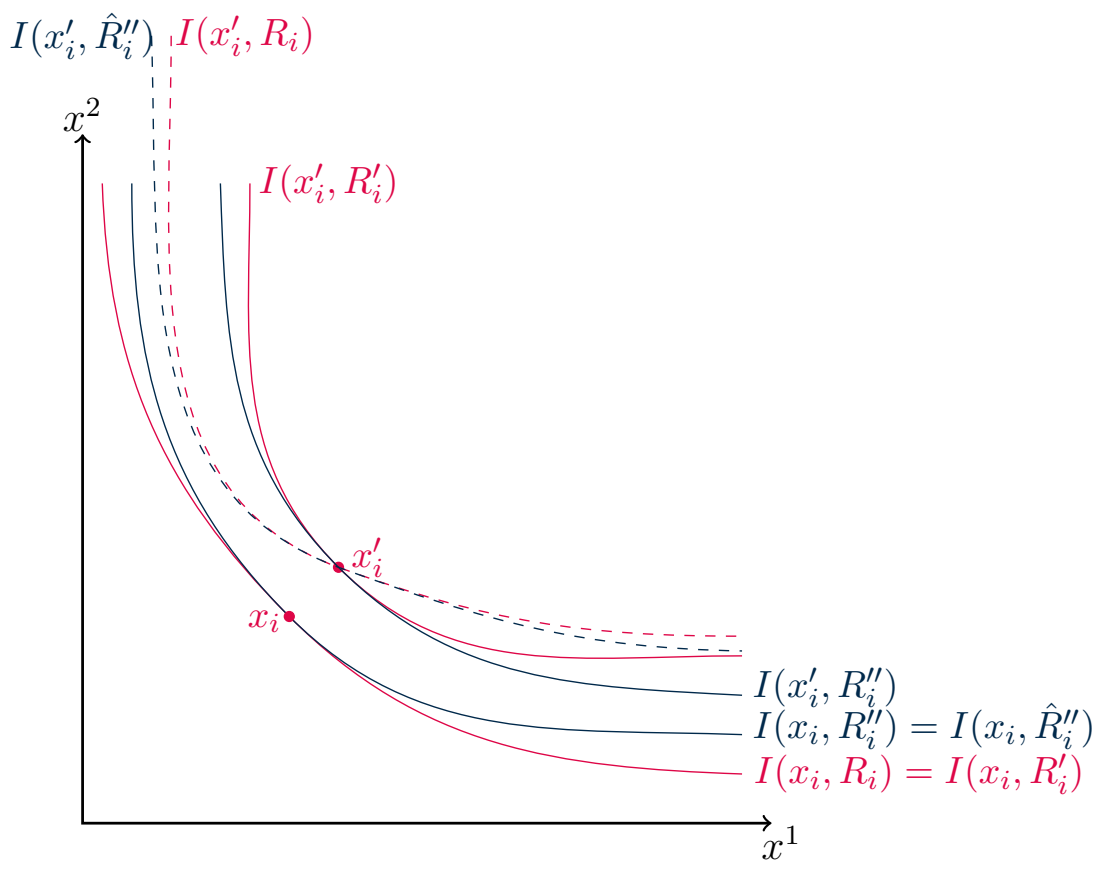

Figure 2: Illustration of the proof of Lemma 1

NotE: The set $I(x, R)$ is the set of bundles that are indifferent to $x$ according to the preference ordering $R$.

Lemma 1 Assume that the social ordering $\succsim$ satisfies Pareto for individual allocation and Preference monotonicity. For all $R_{i}, R_{i}^{\prime} \in \mathcal{R}$ and all $x_{i} \in \mathcal{X}$, if $U\left(x_{i}, R_{i}\right)=U\left(x_{i}, R_{i}^{\prime}\right)$ then $\left(x_{i}, R_{i}\right) \sim\left(x_{i}, R_{i}^{\prime}\right)$.

Proof. Consider $R_{i}, R_{i}^{\prime} \in \mathcal{R}$ and $x_{i} \in \mathcal{X}$ such that $U\left(x_{i}, R_{i}\right)=U\left(x_{i}, R_{i}^{\prime}\right)$. For any $x_{i}^{\prime} \gg x_{i}$, we can find $R_{i}^{\prime \prime}$ such that $U\left(x_{i}, R_{i}^{\prime \prime}\right) \subsetneq U\left(x_{i}, R_{i}\right)$ and $U\left(x_{i}^{\prime}, R_{i}^{\prime}\right) \subsetneq$ $U\left(x_{i}^{\prime}, R_{i}^{\prime \prime}\right)$ (see Figure 2). By Preference monotonicity, $\left(x_{i}, R_{i}^{\prime \prime}\right) \succsim\left(x_{i}, R_{i}\right)$ and $\left(x_{i}^{\prime}, R_{i}^{\prime}\right) \succsim\left(x_{i}^{\prime}, R_{i}^{\prime \prime}\right)$. By Pareto for individual allocation, $\left(x_{i}^{\prime}, R_{i}^{\prime \prime}\right) \succ\left(x_{i}, R_{i}^{\prime \prime}\right)$. By transitivity of $\succsim$, we thus have $\left(x_{i}^{\prime}, R_{i}^{\prime}\right) \succ\left(x_{i}, R_{i}\right)$. By continuity of $\succsim$, we obtain $\left(x_{i}, R_{i}^{\prime}\right) \succsim\left(x_{i}, R_{i}\right) .^{7}$

Similarly (see Figure 2), we can find $\hat{R}_{i}^{\prime \prime}$ such that $U\left(x_{i}, \hat{R}_{i}^{\prime \prime}\right) \subsetneq U\left(x_{i}, R_{i}^{\prime}\right)$ and $U\left(x_{i}^{\prime}, R_{i}\right) \subsetneq U\left(x_{i}^{\prime}, \hat{R}_{i}^{\prime \prime}\right)$ to prove that $\left(x_{i}, R_{i}\right) \succsim\left(x_{i}, R_{i}^{\prime}\right)$. Therefore $\left(x_{i}, R_{i}\right) \sim$

${ }^{7}$ Indeed, we can construct a sequence $\left(x_{i}^{\prime}\right)^{k}$ of allocations such that $\left(x_{i}^{\prime}\right)^{k} \gg x_{i},\left(\left(x_{i}^{\prime}\right)^{k}, R_{i}^{\prime}\right) \succ$ $\left(x_{i}, R_{i}\right)$, and $\left(x_{i}^{\prime}\right)^{k}$ converges to $x_{i}$, using the reasoning above. 
$\left(x_{i}, R_{i}^{\prime}\right)$

Preference monotonicity implies a form of indifference to risk attitudes as far as well-being comparisons are concerned. Indeed, two preference orderings $R_{i}$ and $R_{i}^{\prime}$ that represent different risk attitudes but yield the same ordinal preferences over bundles have the same upper-contour sets as defined here. So Lemma 1 implies that $\left(x_{i}, R_{i}\right) \sim\left(x_{i}, R_{i}^{\prime}\right)$ for all $x_{i} \in \mathcal{X}$ in that case. We revisit this property in Section 5 and examine a weaker version of Preference monotonicity, applied to upper-contour sets on risky prospects rather than sure bundles, which does not imply such indifference to risk attitudes.

Up to now, we have introduced principles that apply in single individual societies. Social comparisons in that case boil down to measuring and comparing individual wellbeing. It seems natural to ground judgements regarding broader collective allocations on the distribution of such measures of individual wellbeing. This justifies to make a link between the assessments of single-individual societies and social allocations. Specifically, John Broome suggested the following principle (Broome, 1991):

Betterness dominance. For all $N \in \mathcal{N}$, all $R_{N}, R_{N}^{\prime} \in \mathcal{R}^{N}$ and all $x_{N}, x_{N^{\prime}} \in \mathcal{X}$, if $\left(x_{i}, R_{i}\right) \succ\left(x_{i}^{\prime}, R_{i}^{\prime}\right)$ for all $i \in N$ then $\left(x_{N}, R_{N}\right) \succ\left(x_{N}^{\prime}, R_{N}^{\prime}\right)$.

In this paper, we shall focus on fair evaluations of allocations. A natural basic fairness principle is Anonymity.

Anonymity. For all $N, M \in \mathcal{N}$ with $|N|=|M|$, for all $\left(x_{N}, R_{N}\right),\left(x_{M}^{\prime}, R_{M}^{\prime}\right) \in \mathcal{A}$, if there exists a bijection $\sigma: N \rightarrow M$ such that for all $i \in N\left(x_{i}, R_{i}\right)=$ $\left(x_{\sigma(i)}^{\prime}, R_{\sigma(i)}^{\prime}\right)$, then $\left(x_{N}, R_{N}\right) \sim\left(x_{M}^{\prime}, R_{M}^{\prime}\right)$.

We henceforth assume that all the principles above are normatively appealing and we name universal social orderings satisfying these principles "fair universal social orderings".

Definition $1 A$ fair universal social welfare ordering is a social welfare ordering $\succsim$ on $\mathcal{F}$ satisfying Pareto for individual allocation, Preference monotonicity, Betterness dominance, and Anonymity. 
The next proposition provides a justification for using well-being measures based on indifference curves by using a set function to measure upper contour sets as explained in Section 2.2. To state the proposition, let us introduce some definitions. A function $W: \mathbb{R}_{+}^{n} \rightarrow \mathbb{R}_{+}$is said to be monotonic when, for any $z$, $z^{\prime} \in \mathbb{R}_{+}^{n}, z \gg z^{\prime}$ implies $W(z)>W\left(z^{\prime}\right)$. It is said to be normalized if for all $t \in \mathbb{R}_{+}, W(t, \cdots, t)=t$. Normalized social welfare functions are useful in welfare economics because they measure social welfare in terms of "equally-distributed equivalent" (EDE), i.e., they indicate the level of individual well-being that, if equally enjoyed by everyone in the population, would yield the same social welfare as the actual distribution.

Proposition 1 If a social welfare ordering $\succsim$ on $\mathcal{F}$ is a fair universal social welfare ordering then there exists a continuous, increasing set function $\psi: \mathcal{U} \rightarrow$ $\mathbb{R}$ and, for each $n \in \mathbb{N}$, a continuous, symmetric, monotonic, and normalized function $W_{n}: \mathbb{R}_{+}^{n} \rightarrow \mathbb{R}_{+}$such that, for all $N, M \in \mathcal{N}$ with $|N|=|M|=n$, for all $\left(x_{N}, R_{N}\right),\left(x_{M}^{\prime}, R_{M}^{\prime}\right) \in \mathcal{A}$ :

$$
\left(x_{N}, R_{N}\right) \succsim\left(x_{M}^{\prime}, R_{M}^{\prime}\right) \Longleftrightarrow W_{n}\left(\left(u_{\psi}\left(x_{i}, R_{i}\right)\right)_{i \in N}\right) \geq W_{n}\left(\left(u_{\psi}\left(x_{j}^{\prime}, R_{j}^{\prime}\right)\right)_{j \in M}\right) .
$$

Proposition 1 is similar to the well-known "welfarism theorem" in social choice and welfare theory (see d'Aspremont and Gevers, 1977). However, the approach departs from classical social choice theory with interpersonally comparable utilities, because the approach relies only on ordinal non-comparable information on preferences and utility numbers are not given as an input to the analysis, but derived from the axiomatization. Specifically, we obtain that individual wellbeing measurement should be based on set functions, and then aggregated in an impartial way.

\subsection{Social welfare measurement and risk: An impossibility result}

Preference monotonicity is a key property in our definition of fair universal social welfare orderings, as it connects to standard well-being measures in fair allocation 
theory. In this section, we show how this principle conflicts with two important principles for social decision making under uncertainty, namely State-wise dominance and a minimal version of ex ante Pareto (i.e., applied to a one-individual society). These two principles are at the core of the stronger requirements used by Harsanyi (1955) in his seminal contribution on welfare evaluation under risk.

State-wise dominance is a requirement of social rationality. It states that whenever a prospect yields a better alternative in all states of the world, it is also better ex ante.

State-wise dominance. For all $f, g \in \mathcal{F}$, if $f(s) \succsim g(s)$ for all $s \in \mathcal{S}$, then $f \succsim g$

State-wise dominance is much weaker than the social expected utility hypothesis used by Harsanyi (1955).

Social expected utility. There exists a function $V: \mathcal{A} \rightarrow \mathbb{R}$, which is unique up to increasing affine transformations, such that, for all $f, g \in \mathcal{F}$ :

$$
f \succsim g \Longleftrightarrow \mathbb{E}[V \circ f] \geq \mathbb{E}[V \circ g]
$$

A second key requirement in Harsanyi (1955) is that individual preferences should be respected. This is the well-known Pareto principle. Given that our framework includes risk on the existence of people composing the population, we may however wonder how the Pareto principle should be applied. Indeed, individual preferences are normatively relevant only for states of the world in which the preference bearers exist. So we may consider the following minimal requirement.

Pareto for individual risk. For all $f, g \in \mathcal{F}$, if there exists $i \in \mathbb{N}$ such that for all $s \in \mathcal{S}, N(f(s))=N(f(s))=\{i\}$ and $R_{i}(f(s))=R_{i}(g(s))=R_{i}$, then $f \succ g$ if $\mathbb{E}\left[v_{R_{i}} \circ x_{i}(f)\right]>\mathbb{E}\left[v_{R_{i}} \circ x_{i}(g)\right]$.

One may want to apply this principle in situations where several persons exist. The problem is that people may not exist for sure with stable preferences. A more general Pareto principle states that individual preferences should be respected 
whenever individuals live in the same states of the world in the two prospects that are being compared. Hence, we propose to apply Pareto conditional on existence in certain states of the world.

Pareto. For all $f, g \in \mathcal{F}$, if $E_{i, R_{i}}(f)=E_{i, R_{i}}(g):=E_{i, R_{i}}$ for all $i \in \mathbb{N}$ and $R_{i} \in \mathcal{R}$, and if $\mathbb{E}_{E_{i, R_{i}}}\left[v_{R_{i}} \circ x_{i}(f)\right]>\mathbb{E}_{E_{i, R_{i}}}\left[v_{R_{i}} \circ x_{i}(g)\right]$ for all $i \in \mathbb{N}$ and $R_{i} \in \mathcal{R}$ such that $P\left(E_{i, R_{i}}\right)>0$, then $f \succ g$.

This Pareto principle is the so-called "Weak Pareto" principle, which is compatible with Maximin.

Our first main result is that, even if we only focus on the very weak requirements of State-wise dominance and Pareto for individual risk, we obtain an impossibility result.

Theorem 1 There is no social welfare ordering $\succsim$ on $\mathcal{F}$ satisfying Preference monotonicity, Pareto for individual risk and State-wise dominance.

Proof. Consider any individual $i$. Let $R_{i}, R_{i}^{\prime} \in \mathcal{R}$ be two preference relations such that for all $x_{i}, x_{i}^{\prime} \in \mathcal{X}, x_{i} R_{i} x_{i}^{\prime} \Longleftrightarrow x_{i} R_{i}^{\prime} x^{\prime}$, but there exists $\ell, \ell^{\prime} \in \mathcal{L}$ such that $\ell I_{i} \ell^{\prime}$ but $\ell P_{i}^{\prime} \ell^{\prime}$. Thus $R_{i}$ and $R_{i}^{\prime}$ induce the same preferences on riskless outcomes but exhibit different risk attitudes.

Consider $f, g, \hat{f}, \hat{g} \in \mathcal{F}$ such that $E_{i, R_{i}}(f)=E_{i, R_{i}}(g)=E_{i, R_{i}^{\prime}}(\hat{f})=E_{i, R_{i}^{\prime}}(\hat{g})=$ $\mathcal{S}$, and, for all $s \in \mathcal{S}, N(f(s))=N(g(s))=N(\hat{f}(s))=N(\hat{g}(s))=\{i\}, x_{i}(f(s))=$ $x_{i}(\hat{f}(s))=\ell(s)$ and $x_{i}(g(s))=x_{i}(\hat{g}(s))=\ell^{\prime}(s)$. I.e., $f, g, \hat{f}, \hat{g}$ are acts for which only $i$ exists, with preferences $R_{i}$ in $f$ and $g$ and with preferences $R_{i}^{\prime}$ in $\hat{f}$ and $\hat{g}$. Furthermore, $f$ and $\hat{f}$ induce the same outcomes as $\ell$, while $g$ and $\hat{g}$ induce the same outcomes as $\ell^{\prime}$. By Pareto for individual risk (and continuity), $f \sim g$ but $\hat{f} \succ \hat{g}$. (Observe that by continuity, Pareto for individual risk implies that individual indifference between two lotteries implies social indifference between them).

By assumption, for any $x_{i} \in \mathcal{X}$, we have $U\left(x_{i}, R_{i}\right)=U\left(x_{i}, R_{i}^{\prime}\right)$. In particular, for all $s \in \mathcal{S}, U\left(x_{i}(f(s)), R_{i}\right)=U\left(x_{i}(\hat{f}(s)), R_{i}^{\prime}\right)$ and $U\left(x_{i}(g(s)), R_{i}\right)=$ $U\left(x_{i}(\hat{g}(s)), R_{i}^{\prime}\right)$. By Lemma 1, given that Pareto for individual risk implies Pareto 
for individual allocation, $f(s) \sim \hat{f}(s)$ and $g(s) \sim \hat{g}(s)$ for all $s \in \mathcal{S}$. State-wise

dominance thus implies that $f \sim \hat{f}$ and $g \sim \hat{g}$. Given that $f \sim g$ and $\hat{f} \succ \hat{g}$ (see above), by transitivity we have a contradiction.

Theorem 1 offers two routes to obtain specific social welfare criteria while maintaining Preference monotonicity: weakening Pareto for individual risk or weakening State-wise dominance. The first route will yield an ex-post approach where individual risk attitudes will not matter. The second route will yield an exante approach that may yield inconsistent social choices. Moreover, a third route would involve weakening Preference monotonicity: we will study this possibility in Section 5.

\section{Fair universal social welfare orderings: ex ante and ex post approaches}

\subsection{Variable population and consistency across population sizes}

As we have mentioned in the introduction, social uncertainty may bear not only on the allocation and preferences of the population, but also on the composition and size in the population. This is particularly true when we design policies that may affect some future people whose numbers may be uncertain. The need for population criteria has then often been felt in the literature on growth with endogenous population (Nerlov, Razin and Sadka, 1985; Palivos and Yip, 1993; Golosov, Jones and Tertilt, 2007; Boucekkine, Fabbri and Gozzi, 2013; Lawson and Spears, 2018) and the literature on climate change mitigation (Millner, 2013). Average and total welfare have been popular criteria while the literature on population ethics has popularized critical-level utilitarianism as an alternative (Broome, 2004; Blackorby, Bossert and Donaldson, 2005).

In that context, a basic principle is that we can make trade-offs between population size and total welfare or, said differently, that we can compare population with different sizes in a non-trivial way. This is ensured by the following principle. 
Indifferent addition. For all $N \in \mathcal{N}$ and all $\left(x_{N}, R_{N}\right) \in(\mathcal{X} \times \mathcal{R})^{N}$, there exist $i \notin N, x_{i} \in \mathcal{X}$ and $R_{i} \in \mathcal{R}$ such that $\left(x_{N}, R_{N}\right) \sim\left(\left(x_{N}, R_{N}\right),\left(x_{i}, R_{i}\right)\right)$.

Indifferent addition is very general principle that merely requires that it is always possible to add a new individual to the population without changing aggregate social welfare. Our weak formulation does not put any constraint on the characteristics of the admissible new individual, which may vary depending on the size, preference profile and allocation of the incumbent population.

The next two principles are requirements of consistency when comparing populations with different sizes. The first principle is Extended replication invariance, as proposed by Zoli (2009). It is related to the Population replication principle, introduced in Bossert (1990) and based on Dalton's (1920) "principle of proportionate additions to persons". It requires welfare comparisons to be invariant with respect to replications of the population.

To introduce Extended replication invariance, let us first introduce the notion of a $k$-replica, with $k \in \mathbb{N}$. For any $\left(x_{N}, R_{N}\right) \in \mathcal{A},\left(x_{N^{\prime}}^{\prime}, R_{N^{\prime}}^{\prime}\right) \in \mathcal{A}$ is a $k$-replica of $\left(x_{N}, R_{N}\right)$ if $\left|N^{\prime}\right|=k|N|$, and there exists a partition $\left(M_{1}, \cdots, M_{|N|}\right)$ of $N^{\prime}$ into $|N|$ groups of size $k$ and a bijection $\sigma: N \rightarrow\{1, \cdots,|N|\}$ such that, for all $i \in N$ and all $j \in M_{\sigma(i)},\left(x_{j}^{\prime}, R_{j}^{\prime}\right)=\left(x_{i}, R_{i}\right)$.

Extended replication invariance. For all $f, g, f^{\prime}, g^{\prime} \in \mathcal{F}$, if there exists $k \in \mathbb{N}$ such that for all $s \in \mathcal{S}, f^{\prime}(s)$ is a $k$-replica of $f(s)$ and $g^{\prime}(s)$ is a $k$-replica of $g(s)$, then $f^{\prime} \succsim g^{\prime}$ if and only if $f \succsim g$.

Such a replication invariance principle implies that, when comparing prospects with the same population size, the social evaluation only depends on the distribution of well-being, while for comparisons across prospects with different population sizes, only the relative population sizes may matter.

Another principle is Independence of the sure. The idea behind the principle is that the social ranking of alternatives should not depend on the situation (and well-being) of people who are not affected by the decision. Specifically, the principle says that the social evaluation should not depend on the situation of those individuals who exist for sure and face no risk. The restriction to individuals 
facing no risk is motivated by the fact that people who are not affected by the decision but whose fate is correlated with others may influence ex post inequalities and be relevant to the evaluation of prospects.

Independence of the sure. For all $f, g, f^{\prime}, g^{\prime}, \hat{f}, \hat{g} \in \mathcal{F}$ if there exist $N \in \mathcal{N}$, $\left(x_{N}, R_{N}\right),\left(x_{N}^{\prime}, R_{N}^{\prime}\right) \in(\mathcal{X} \times \mathcal{R})^{N}$ such that for all $s \in \mathcal{S}, N(f(s)) \cap N=$ $\emptyset, N(g(s)) \cap N=\emptyset, f^{\prime}(s)=\left(f(s),\left(x_{N}, R_{N}\right)\right), g^{\prime}(s)=\left(g(s),\left(x_{N}, R_{N}\right)\right)$, $\hat{f}(s)=\left(f(s),\left(x_{N}^{\prime}, R_{N}^{\prime}\right)\right)$ and $\hat{g}(s)=\left(g(s),\left(x_{N}^{\prime}, R_{N}^{\prime}\right)\right)$, then $f^{\prime} \succsim g^{\prime}$ if and only if $\hat{f} \succsim \hat{g}$.

A similar but stronger principle is the principle of Separability proposed by Fleurbaey and Tadenuma (2014). It claims that removing these people from the population would not affect the social evaluation. Independence of the sure is also related to Independence of the utility of the dead (Blackorby, Bossert and Donaldson, 2002), which states that we can ignore the well-being level of dead people existing in the past.

\subsection{Ex-post criteria}

As mentioned before, Theorem 1 suggests two ways to obtain social welfare criteria satisfying Preference monotonicity: weakening Pareto for individual risk or weakening State-wise dominance. In this section, we explore ex-post criteria that are based on well-defined ex-post social evaluations which are aggregated ex ante in a consistent way, satisfying State-wise dominance (and even Social expected utility). To do so, we have to drop any form of the ex ante Pareto principle, although we keep the ex post Pareto principle contained in the definition of a fair universal social ordering.

To introduce our main results, we need additional definitions. Let $\mathcal{Z}=$ $\cup_{n \in \mathbb{N}} \mathbb{R}_{+}^{n}$ be the set of all finite vectors of non-negative numbers. We use the same vocabulary as above and for any $n, k \in \mathbb{N}$, any $z \in \mathbb{R}_{+}^{n}$, we say that $z^{\prime} \in \mathbb{R}_{+}^{k n}$ is a $k$ -

replica of $z$ if for all $i \in\{1, \cdots, n\}$ and $j \in\{1, \cdots, k\}, z_{k(i-1)+j}^{\prime}=z_{i}$. A function $W: \mathcal{Z} \rightarrow \mathbb{R}$ is said to be replication-invariant if for all $z, z^{\prime} \in \mathcal{Z}, W(z)=W\left(z^{\prime}\right)$ 
whenever there exists $k \in \mathbb{N}$ such that $z^{\prime}$ is a $k$-replica of $z$. For a function $\varphi: \mathbb{R}_{+} \rightarrow \mathbb{R}$ and for any $n \in \mathbb{N}$ we denote $D_{\varphi}^{n}=\left\{t \in \mathbb{R} \mid \exists z \in \mathbb{R}_{+}^{n}, t=\sum_{i=1}^{n} \varphi\left(z_{i}\right)\right\}$.

Theorem 2 If a fair universal social welfare ordering $\succsim$ satisfies Indifferent addition and Social expected utility, then there exists a continuous and increasing set function $\psi$, a function $V: \mathbb{N} \times \mathbb{R}_{+} \rightarrow \mathbb{R}$, continuous and increasing in its second argument, and for each $n \in \mathbb{N}$ a continuous, symmetric, monotonic, and normalized function $W_{n}: \mathbb{R}_{+}^{n} \rightarrow \mathbb{R}_{+}$such that $\succsim$ is represented by the function:

$$
\mathbb{E}\left[V\left(n(f), W_{n(f)}\left(\left(u_{\psi}\left(x_{i}(f), R_{i}(f)\right)\right)_{i \in N(f)}\right)\right)\right]
$$

If furthermore:

1. ¿ satisfies Extended replication invariance, then there is a replication-invariant function $W: \mathcal{Z} \rightarrow \mathbb{R}_{+}$such that $W_{n}=W$ for all $n \in \mathbb{N}$, and there exist $\alpha, \beta \in \mathbb{R}$ such that :

$$
V(n, w)=\left\{\begin{array}{l}
n^{\alpha}(w-\beta) \text { if } \alpha \neq 0 \\
w+\beta \ln (n) \text { if } \alpha=0
\end{array}\right.
$$

2. $\succsim$ satisfies Independence of the sure, then there exists a continuous and increasing function $\varphi: \mathbb{R}_{+} \rightarrow \mathbb{R}$ such that for all $n \in \mathbb{N}$ and $z \in \mathbb{R}_{+}^{n}$, $W_{n}(z)=\varphi^{-1}\left(\frac{1}{n} \sum_{i=1}^{n} \varphi\left(z_{i}\right)\right)$, and there exist $\alpha \in \mathbb{R}$ and for each $n \in \mathbb{N}, a$ constant $c_{n} \in D_{\varphi}^{n}$ such that

$$
V(n, w)=\left\{\begin{array}{l}
n \varphi(w)-c_{n} \text { if } \alpha=0 \\
\frac{1}{\alpha} e^{\alpha\left(n \varphi(w)-c_{n}\right)} \text { if } \alpha \neq 0
\end{array}\right.
$$

Theorem 2 indicates that ex-ante social welfare criteria take the expected value of (ex-post) variable population universal social welfare orderings, whose general form is similar to that described in Theorem 1 of Fleurbaey and Tadenuma (2014). Comparisons of individual levels of well-being are based on set functions. Then the measures of individual well-being are aggregated through a social welfare 
function $W_{n}$ which may depend on population size. And last, these social welfare measures are combined with population size to obtain overall social welfare.

Points 1 and 2 of Theorem 2 provides specific social welfare formulas when Extended replication invariance or Independence of the sure are satisfied. Quite naturally, Extended replication invariance implies replication invariant fixed-population social welfare criteria: social welfare only depends on the distribution of individual well-being when population size is fixed. More interestingly, Extended replication invariance also implies constraints on how population with different sizes are compared: either we multiply population size to the power $\alpha$ with the difference between the EDE level of individual well-being and a critical value $\beta$ (setting the 0 level for egalitarian populations); or population size brings additional welfare $\beta \ln (n)$ that increases logarithmically. The first possibility has two interesting special cases: when $\alpha=1$ we have a "total" approach to social welfare, with critical-level (similar to critical-level utilitarianism as described in Blackorby, Bossert and Donaldson, 2005); when $\alpha=0$ we have an "average" type of criterion (similar to average utilitarianism as described in Blackorby, Bossert and Donaldson, 2005).

When Independence of the sure is satisfied, we obtain specific transformations of social criteria that are formally similar to critical-level utilitarianism (Blackorby, Bossert and Donaldson, 2005). We thus have very specific variable population criteria, although Independence of the sure is a rather weak separability principle. The affine or exponential form of the transformation of critical-level utilitarianism is similar to the one obtained in Bommier and Zuber (2008). When $\alpha<0$, we obtain a concave transformation of total utility, and $-\alpha$ can be interpreted as risk aversion with respect to social welfare, or as an aversion to catastrophic risks.

\subsection{Ex-ante criteria}

Ex ante criteria satisfy the Pareto principle presented in Section 3.2. We know by Theorem 1 that in that case, we cannot keep expected utility for the social criterion. However, it is desirable to retain as much of social rationality as possi- 
ble. To do so, we assume that social judgements are based on an expected utility for the subset of prospects such that people do not face any risk conditional on existing with particular preferences. Such prospects can be viewed as prospects involving only a social risk on the composition (number of people and preferences) of the population. Let us denote this set of prospects $\overline{\mathcal{F}}$. The formal definition of $\overline{\mathcal{F}}$ is

$$
\overline{\mathcal{F}}=\left\{f \in \mathcal{F} \mid \forall i \in \mathbb{N}, \forall R_{i} \in \mathcal{R}, x_{i}(f(s))=x_{i}\left(f\left(s^{\prime}\right)\right) \text { for all } s, s^{\prime} \in E_{i, R_{i}}(f)\right\}
$$

Restricted social expected utility. There exists a function $V: \mathcal{A} \rightarrow \mathbb{R}$, unique up to an increasing affine transformation, such that, for all $f, g \in \overline{\mathcal{F}}$ :

$$
f \succsim g \Longleftrightarrow \mathbb{E}[V(f)] \geq \mathbb{E}[V(g)]
$$

We obtain the next result.

Theorem 3 If a fair universal social welfare ordering $\succsim$ satisfies Indifferent addition, Pareto and Restricted social expected utility, then there exists a continuous and increasing set function $\psi$, a function $V: \mathbb{N} \times \mathbb{R}_{+} \rightarrow \mathbb{R}$, continuous and increasing in its second argument, and for each $n \in \mathbb{N}$ a continuous, symmetric, monotonic, and normalized function $W_{n}: \mathbb{R}_{+}^{n} \rightarrow \mathbb{R}_{+}$such that $\succsim$ is represented by the function: :

$$
\sum_{\left(N, R_{N}\right) \in\left(N, R_{N}\right)(f(\mathcal{S}))} p_{N, R_{N}}(f) V\left(|N|, W_{|N|}\left(\left(u_{\psi}\left(c e_{i, R_{i}}(f), R_{i}\right)\right)_{i \in N}\right)\right) .
$$

If furthermore:

1. ¿ satisfies Extended replication invariance, then there is a replication-invariant function $W: \mathcal{Z} \rightarrow \mathbb{R}_{+}$such that $W_{n}=W$ for all $n \in \mathbb{N}$, and there exist $\alpha, \beta \in \mathbb{R}$ such that :

$$
V(n, w)=\left\{\begin{array}{l}
n^{\alpha}(w-\beta) \text { if } \alpha \neq 0 \\
w+\beta \ln (n) \text { if } \alpha=0
\end{array}\right.
$$


2. $\succsim$ satisfies Independence of the sure, then there exists a continuous and increasing function $\varphi: \mathbb{R}_{+} \rightarrow \mathbb{R}$ such that for all $n \in \mathbb{N}$ and $z \in \mathbb{R}_{+}^{n}$, $W_{n}(z)=\varphi^{-1}\left(\frac{1}{n} \sum_{i=1}^{n} \varphi\left(z_{i}\right)\right)$, and there exist $\alpha \in \mathbb{R}$ and for each $n \in \mathbb{N}$, a constant $c_{n} \in D_{\varphi}^{n}$ such that

$$
V(n, w)=\left\{\begin{array}{l}
n \varphi(w)-c_{n} \text { if } \alpha=0 \\
\frac{1}{\alpha} e^{\alpha\left(n \varphi(w)-c_{n}\right)} \text { if } \alpha \neq 0
\end{array}\right.
$$

The construction of social welfare in Theorem 3 is the following. First, we compute the certainty-equivalent bundle (conditional on existing with certain preferences) for each individual. We can associate this certainty-equivalent bundle to a level of well-being $\left.u_{\psi}\left(c e_{i, R_{i}}(f), R_{i}\right)\right)_{i \in N}$ in states of the worlds where individual $i$ exists with preferences $R_{i}$. We aggregate these well-being levels (rather than the actual ones) in each state of the world and then take an expected value of these aggregate social welfare levels, which also take into account population size. Hence, states of the world where the same population exists with particular preferences yield the same social welfare level after subsituting the certainty-equivalent bundles for the actual ones, which justifies using the probability weights $p_{N, R_{N}}(f)$.

A key observation is that the ex-ante social welfare criteria of Theorem 3 are exactly the same as the ex-post social welfare criteria of Theorem 2 for acts where individuals bear no risk on consumption, for given preferences. Hence, if we can ignore the consumption risk individuals face, there is no difference between the ex-ante and the ex-post approach. The only difference is that, for risk on consumption, we use certainty-equivalent bundles to account for individuals' aversion to risk in the ex-ante approach (whereas we completely ignore those individual risk attitudes in the ex-post approach).

This observation explains why we obtain similar specific criteria for variable population comparisons in Theorems 2 and 3 when we add Extended replication invariance and Independence of the sure. 


\section{$5 \quad$ Weakening Preference monotonicity}

Theorem 1 highlighted the impossibility to combine three principles: Preference monotonicity, Pareto for individual risk, and State-wise dominance. We have explored criteria satisfying Preference monotonicity but weakening one of the other two principles. However, one may also want to try to weaken Preference monotonicity.

To do so, let us introduce an additional definition. For any $x_{i} \in \mathcal{X}$ and $R_{i} \in \mathcal{R}$, we denote $\bar{U}\left(x_{i}, R_{i}\right)=\left\{f_{i}: S \rightarrow \mathcal{X} \mid f_{i} R_{i} x_{i}\right\}$ the upper-contour set of $x_{i}$ according to $R_{i}$, extended to include lotteries. A weakening of Preference monotonicity is:

Weak preference monotonicity. For all $i \in \mathbb{N}$ and all $R_{i}, R_{i}^{\prime} \in \mathcal{R}$, for any $x_{i} \in \mathcal{X}$, if $\bar{U}\left(x_{i}, R_{i}\right) \subsetneq \bar{U}\left(x_{i}, R_{i}^{\prime}\right)$ then $\left(x_{i}, R_{i}\right) \succsim\left(x_{i}, R_{i}^{\prime}\right)$.

Weak preference monotonicity can take into account risk preference when measuring utility. Indeed, we know that, when individuals have preferences over several commodities, comparisons of risk aversion are possible in a systematic way only when individuals have the same ordinal preferences over commodities Kihlstrom and Mirman (1974). But we also know that in that case, at a sure bundle, a more risk averse individual always has an upper-contour set above the upper-contour set of a less risk averse individual. This is precisely the definition of more risk averse given by Kihlstrom and Mirman (1974). Weak preference monotonicity thus implies that a more risk averse individual always has a higher level of well-being at any sure allocation: risk preferences are thus taken into account in wellbeing measurement.

However, weakening Preference monotonicity in this way is not enough to escape our initial impossibility result.

Proposition 2 There is no social welfare ordering $\succsim$ on F satisfying Weak preference monotonicity, Pareto for individual risk and Social expected utility.

Proof. By Social expected utility, there exists a function $V: \mathcal{A} \rightarrow \mathbb{R}$, which is unique up to increasing affine transformations, such that, for all $f, g \in F: f \succsim$

$g \Longleftrightarrow \mathbb{E}[V \circ f] \geq \mathbb{E}[V \circ g]$. By Pareto for individual risk, for all $f, g \in F$, if there 
exists $i \in \mathbb{N}$ such that $N(f(s))=N(g(s))=\{i\}$ and $R_{i}(f(s))=R_{i}(g(s))=R_{i}$ for all $s \in S$, then $f \succ g$ whenever $\mathbb{E}\left[v_{R_{i}}\left(x_{i}(f)\right)\right]>\mathbb{E}\left[v_{R_{i}}\left(x_{i}(g)\right)\right]$. Hence, for any $i \in \mathbb{N}$ and $R_{i} \in \mathcal{R}$, there exists $\alpha_{R_{i}}^{i} \in \mathbb{R}_{++}$and $\beta_{R_{i}}^{i} \in \mathbb{R}$ such that $V\left(x_{i}, R_{i}\right)=\alpha_{R_{i}}^{i} v_{R_{i}}\left(x_{i}\right)+\beta_{R_{i}}^{i}$ for all $x_{i} \in R_{+}^{\ell}$.

Let $\omega \in \mathcal{X} \backslash\{0\}$ be a given reference bundle. Consider $R, R_{i}, R_{i}^{\prime} \in \mathcal{R}$ such that $v_{R_{i}}(x)=e_{\omega}(x, R)$ and $v_{R_{i}^{\prime}}(x)=1-\left[e_{\omega}(x, R)\right]^{-1}$ for any $x \in \mathcal{X}$. Given that $v_{R_{i}^{\prime}}$ is an increasing concave transformation of $v_{R_{i}}$, we know (Kihlstrom and Mirman, 1974, Prop. 6) that for any $x_{i} \in \mathcal{X}, \bar{U}\left(x_{i}, R_{i}^{\prime}\right) \subset \bar{U}\left(x_{i}, R_{i}\right)$. By Weak preference monotonicity, we should have $\left(x_{i}, R_{i}^{\prime}\right) \succsim\left(x_{i}, R_{i}\right)$.

However, we have seen before that there exists $\alpha_{R_{i}}^{i}, \hat{\alpha}_{R_{i}^{\prime}}^{i} \in \mathbb{R}_{++}$and $\beta_{R_{i}}^{i}, \hat{\beta}_{R_{i}^{\prime}}^{i} \in$ $\mathbb{R}$ such that $V\left(x_{i}, R_{i}\right)=\alpha_{R_{i}}^{i} v_{R_{i}}\left(x_{i}\right)+\beta_{R_{i}}^{i}$ and $V\left(x_{i}, R_{i}^{\prime}\right)=\hat{\alpha}_{R_{i}^{\prime}}^{i} v_{R_{i}^{\prime}}\left(x_{i}\right)+\hat{\beta}_{R_{i}^{\prime}}^{i}$. To satisfy Weak preference monotonicity, we should thus have, for any $x \in \mathcal{X}$ :

$$
1-\left[e_{\omega}(x, R)\right]^{-1} \geq \frac{\alpha_{R_{i}}^{i}}{\hat{\alpha}_{R_{i}^{\prime}}^{i}} e_{\omega}(x, R)+\frac{\beta_{R_{i}}^{i}-\hat{\beta}_{R_{i}^{\prime}}^{i}}{\hat{\alpha}_{R_{i}^{\prime}}^{i}} .
$$

This is not possible as the left hand side is bounded above on $\mathcal{X}$ while the right hand side is not. We thus obtain a contradiction.

Proposition 2 suggests that we cannot keep any form of preference monotonicity if we insist on Pareto for individual risk and Social expected utility. Actually, the proof of Proposition 2 shows that, in that case, comparisons of individual levels of wellbeing should be based on VNM utility functions $v_{R_{i}}$, provided they are appropriately scaled. The approach suggested by Harsanyi's (1955), based on a utilitarian sum of VNM utility functions, is of course the prime example, although the issue of utility scaling was not solved in that paper and has remained debated ever since.

But there are other possibilities than utilitarianism. For instance, one could use a line of reasoning similar to the proof of Theorem 2 to characterize universal social orderings $\succsim$ satisfying Pareto for individual risk, Betterness dominance, Anonymity, and Social expected utility. They are such that for each $n \in \mathbb{N}$, there exists a continuous, monotonic and symmetric function $W_{n}: \mathbb{R}^{n} \rightarrow \mathbb{R}$ such that 
$\succsim$ is represented by:

$$
\mathbb{E}\left[W_{n(f)}\left(\left(\tilde{v}_{R_{i}(f)}\left(x_{i}(f)\right)\right)_{i \in N(f)}\right)\right] .
$$

where for each $R \in \mathcal{R}, \tilde{v}_{R}$ is a chosen VNM utility function.

One could actually strengthen Pareto. Fleurbaey (2010) argued that we should respect unanimous preferences in the context of egalitarian acts, i.e., acts such that risk takes a pure macroeconomic form and does not generate ex post inequalities. The rationale is that, in that case, individual ex ante assessments of prospects do not obscure ex post inequality consequences for society. The question, however, is how to define a situation of equality in our context.

Fortunately, given a social welfare ordering $\succsim$, there is a natural way to do so since this ordering is able to compare individual situations. Namely, an egalitarian allocation $a=\left(x_{N}, R_{N}\right) \in \mathcal{A}$ is such that, for all $i, j \in N,\left(x_{i}, R_{i}\right) \sim\left(x_{j}, R_{j}\right)$. From this, we can derive the notion of an egalitarian act as one such that, in each state of the world, an egalitarian allocation obtains. Formally, an act $f \in \mathcal{F}$ is an egalitarian act if, for all $s \in S$, for all $i, j \in N(f(s)),\left(x_{i}(f(s)), R_{i}(f(s))\right) \sim$ $\left(x_{j}(f(s)), R_{j}(f(s))\right)$. Let us denote $\mathcal{F}_{\succsim}^{e}$ the set of egalitarian acts. We propose the following version of Pareto for equal risk as suggested by Fleurbaey (2010), but adapted to our setting:

Pareto for equal risk. For all $f, g \in \mathcal{F}_{\succsim}^{e}$, if $E_{i, R_{i}}(f)=E_{i, R_{i}}(g):=E_{i, R_{i}}$ for all $i \in \mathbb{N}$ and $R_{i} \in \mathcal{R}$, and if $\mathbb{E}_{E_{i, R_{i}}}\left[v_{R_{i}} \circ x_{i}(f)\right]>\mathbb{E}_{E_{i, R_{i}}}\left[v_{R_{i}} \circ x_{i}(g)\right]$ for all $i \in \mathbb{N}$ and $R_{i} \in \mathcal{R}$ such that $P\left(E_{i, R_{i}}\right)>0$, then $f \succ g$.

This principle is satisfied together with Betterness dominance, Anonymity, and Social expected utility by a large class of social welfare orderings. More precisely, we obtain the following result.

Theorem 4 If a universal social welfare ordering $\succsim$ satisfies Betterness dominance, Anonymity, Indifferent addition, Pareto for equal risk and Social expected utility, then for each $n \in \mathbb{N}$ there exist a continuous, symmetric, monotonic, and normalized function $W_{n}: \mathbb{R}_{+}^{n} \rightarrow \mathbb{R}_{+}, \alpha_{n} \in \mathbb{R}_{++}$and $\beta_{n} \in \mathbb{R}$ such that $\succsim$ is 
represented by:

$$
\mathbb{E}\left[\alpha_{n(f)} \times W_{n(f)}\left(\left(\tilde{v}_{R_{i}(f)}\left(x_{i}(f)\right)\right)_{i \in N(f)}\right)+\beta_{n(f)}\right]
$$

where for each $R \in \mathcal{R}, \tilde{v}_{R}$ is a chosen VNM utility function.

If furthermore:

1. ¿ satisfies Extended replication invariance, then there is a replication-invariant function $W: \mathcal{Z} \rightarrow \mathbb{R}_{+}$such that $W_{n}=W$ for all $n \in \mathbb{N}$, and there exist $\alpha, \beta \in \mathbb{R}$ such that :

$$
\alpha_{n} w+\beta_{n}=\left\{\begin{array}{l}
n^{\alpha}(w-\beta) \text { if } \alpha \neq 0 \\
w+\beta \ln (n) \text { if } \alpha=0
\end{array}\right.
$$

2. $\succsim$ satisfies Independence of the sure, then for each $n \in \mathbb{N}, W_{n}=\frac{1}{n} \sum_{i=1}^{n} z_{i}$ and there is a constant $c_{n} \in \mathbb{R}_{+}$such that:

$$
\alpha_{n} w+\beta_{n}=n w-c_{n}
$$

A common feature of all the social welfare criteria discussed in this section is that they make comparisons of individual well-being based on VNM utility functions using a specific normalization $\tilde{v}_{R}$. This is indeed an implication of the combination of Social expected utility and Pareto for individual risk (see the proof of Proposition 2). Even if we accept that VNM utility functions are the right metric of individual well-being, there still remains the question of the right scaling is for these functions. A prominent proposal called "relative utilitarianism" consists in scaling the utility functions so that the utility of a "good" option (generally the best) is equal to one and the utility of a "bad" option (generally the worst) is equal to zero (Dhillon and Mertens, 1999). Fleurbaey and Zuber (2021) propose instead to equalize marginal utilities at the poverty line. The principles we have used in this paper are compatible with these various scaling approaches. 


\section{Conclusion}

In this paper, we have extended the theory of welfare to situations in which uncertainty bears not only on future consumption but also on the preferences and existence of future people. A key feature of this project was to preserve the possibility to rely on the theory of fair social choice for interpersonal comparisons and inequality assessment in final consequences. If such a possibility were barred, this would jeopardize the relevance of the large literature on fair allocation in riskless settings, which has offered valuable suggestions, in particular with the equivalence approach, for measuring advantage and well-being in a way that respects individual preferences without needing any more information about well-being than economic bundles and ordinal non-comparable preferences (about sure bundles).

This endeavor can be described as a mixed success, or mixed failure. The initial impossibility laid out in section 3.2 imposed a choice between social rationality and respect for risk attitudes that appeared starker than in previous literature. Exploring the two horns of the dilemma, we have characterized a class of ex post welfare criteria in which individuals' risk attitudes are completely ignored, although preferences over final consequences are duly respected, and a class of ex ante criteria in which the fair-type measures of well-being were applied to certainty-equivalent bundles. While both classes make it possible to rely on fair allocation theory for the measurement of individual advantage and interpersonal comparisons, they are rather extreme in their ignorance of individual risk attitudes (for the ex post class) or their violation of social rationality (i.e., violating State-wise dominance, for the ex ante class).

The negative message from the initial impossibility is that the compromise proposed by Fleurbaey and Zuber (2017), allowing individual risk attitudes to remain relevant for prospects that do not generate any inequality, as assessed by fair-type measures of well-being, within the framework of a fully rational expected value approach to social welfare, was now out of reach. In section 5 we have shown that allowing such minimal role for individual risk attitudes required abandoning the fair-type measures of well-being, and settling for measures that 
directly come from VNM utility functions, suitably scaled. The reason why the compromise negotiated in Fleurbaey and Zuber (2017) is no longer possible is that in the current framework, preferences can vary from one prospect to the other, requiring comparisons of welfare to be more encompassing than when the population is fixed and has fixed preferences.

We think that the three approaches characterized in this paper all deserve careful consideration. There is one interesting difference between the first two (ex post and ex ante) approaches and the third (VNM-based) one. The latter derives a good part of the social attitude to risk from individuals' attitudes and from the scaling of VNM utilities. Indeed, for prospects in which in every state of the world, the VNM utilities are equal across the existing population, and in which the population size is fixed, social evaluation boils down to the expected value of this egalitarian VNM value. Only the risk about population size requires some external input through the parameter $\alpha_{n}, \beta_{n}$ which cannot in any way be derived from individual attitudes. In contrast, the first two approaches have a social utility function $V$ that embodies social risk attitudes and does not in any way derive from individuals' attitudes. In particular, in the ex ante approach, it serves to assess risks about preferences and about the size of the population, and these are risks over which the individuals' attitudes are not defined in our framework. Our results say nothing about how to chose this $V$ function, and further refinement of the theory is needed in this respect.

How can society form its own risk attitude about risks over which no individual can have a personal, self-centered preference? Social choice is usually conceived as a problem of aggregating individual values and preferences, but there are social issues and risks which have no counterpart at the individual level. Simply declaring that the population can have political views over the fate of humanity and that we should then consider how to synthesize such views is a lazy response, because it does not help the population to form such political preferences in the first place, a task that social welfare theory is supposedly designed to assist. We leave this important and intriguing issue for future research. 


\section{References}

Aczél, J. (1966). Lecture on Functional Equations and their Applications, Academic Press.

Anthoff, D., Tol, R. S. J., Yohe, G. W. (2009). "Risk Aversion, Time Preference and the Social Cost of Carbon", Environmental Research Letter, 4, 024002.

Atkinson, G., Dietz, S., Helgeson, J., Hepburn, C., Saelen, H. (2009). "Siblings, Not Triplets: Social Preferences for Risk, Inequality and Time in Discounting Climate Change", Economics: The Open-Access, Open-Assessment E-Journal, Vol. 3, 200926.

Ayong Le Kama, A. (2001). "Preservation and Uncertain Future Preferences", Economic Theory, 8, 745-752.

Ayong Le Kama, A., Schubert, K (2004). "Growth, Environment and Uncertain Future Preferences", Environmental and Resource Economics, 28, 31-53.

Beltratti, A., Chichilnisky, G., Heal, G. (1998). "Uncertain Future Preferences and Conservation." In G. Chichilnisky, G. Heal, and A. Vercelli (Eds.) Sustainability: Dynamics and Uncertainty, Amsterdam: Kluwer Academic Publishers, chapter 3.4, pp. 257-276.

Blackorby, C., Bossert, W., Donaldson, D. (2001). "Population ethics and the existence of value functions", Journal of Public Economics, 82, 301-308.

Blackorby, C., Bossert, W., Donaldson, D. (2002). "Utilitarianism and the theory of justice". In Arrow K, Sen A, Suzumura K (eds) Handbook of social choice and welfare, vol 2. Elsevier, Amsterdam, pp 543-596.

Blackorby, C., Bossert, W., Donaldson, D. (2005). Population Issues in Social Choice Theory, Welfare Economics, and Ethics, Cambridge: Cambridge University Press.

Bommier, A., Zuber, S. (2008). "Can preferences for catastrophe avoidance reconcile social discounting with intergenerational equity?", Social Choice and Welfare, 31, $415-434$.

Bossert, W. (1990). "Social evaluation with variable population size: an alternative concept", Mathematical Social Sciences, 19, 143-158. 
Boucekkine, R., Fabbri, G., Gozzi F. (2013). "Egalitarianism under population change: Age structure does matter," Journal of Mathematical Economics, 55, 86-100.

Broome, J. (1991). Weighing Goods. Equality, Uncertainty and Time. Oxford: Blackwell.

Broome, J. (2004). Weighing Lives. Oxford: Oxford University Press.

Chambers, C.P., Echenique, E., 2012. When does aggregation reduce risk aversion? Games and Economic Behavior, 76, 582-595.

Dalton, H. (1920). "The measurement of the inequality of incomes", Economic Journal, $20,348-361$.

Dasgupta, P.S. (2008). "Discounting climate change", Journal of Risk and Uncertainty, $37,141-169$.

D'Aspremont, C., Gevers, L. (1977). "Equity and the informational basis of collective choice", ReviewofEconomicStudie, 44, 199-209.

Dhillon, A., Mertens, J.-F. (1999). "Relative utilitarianism: An improved axiomatization", Econometrica, 67, 471-498.

Diamond, P.A. (1967). "Cardinal welfare, individualistic ethics, and interpersonal comparison of utility: Comment", Journal of Political Economy, 75, 765-766.

Epstein, L.G., Segal, U. (1992). "Quadratic social welfare functions", Journal of Political Economy, 100, 691-712.

Fleurbaey, M. (2010). "Assessing risky social situations", Journal of Political Economy, 118, 649-680.

Fleurbaey, M., Maniquet, F. (2011). A Theory of Fairness and Social Welfare, Cambridge: Cambridge University Press.

Fleurbaey, M., Suzumura, K., Tadenuma, K. (2005). "Arrovian aggregation in economic environments: how much should we know about indifference surfaces?", Journal of Economic Theory, 124, 22-44.

Fleurbaey, M., Tadenuma, K. (2014). "Universal social orderings", Review of Economic Studies, 81, 1071-1101.

Fleurbaey, M., Zuber, S. (2017). "Fair management of social risks", Journal of Economic Theory, 169, 666-706. 
Fleurbaey, M., Zuber, S. (2021). "Fair utilitarianism", forthcoming in American Economic Journal: Microeconomics.

Golosov, M., Jones, L.E., Tertilt, M. (2007). "Efficiency with Endogenous Population Growth", Econometrica, 75, 1039-1071.

Harsanyi, J. (1955). "Cardinal welfare, individualistic ethics and interpersonal comparisons of utility," Journal of Political Economy, 63, 309-321.

Hansson, B. (1973). "The independence condition in the theory of social choice", Theory and Decision, 4, 25-49.

Intergovernmental Panel on Climate Change (2014). Climate Change 2014: Mitigation of Climate Change, Cambridge: Cambridge University Press.

Karni, E. (2003). "Impartiality and interpersonal comparisons of variations in wellbeing," Social Choice and Welfare, 21, 95-111.

Kihlstrom, R.E., Mirman, L.J. (1974). "Risk aversion with many commodities," Journal of Economic Theory, 8, 361-388.

Lawson N., Spears D. (2018). "Optimal population and exhaustible resource constraint", Journal of Population Economics, 31, 295-335.

Millner, A. (2013). "On welfare frameworks and catastrophic climate risks", Journal of Environmental Economics and Management, 65, 310-325.

Nerlov M., Razin A., Sadka E. (1985). "Population Size: Individual Choice and Social Optima", The Quarterly Journal of Economics, 100, 321-334.

Radó F., Baker J. (1987). "Pexider's equation and aggregation of allocations", Aequationes Mathematicae, 32, 227-239.

Palivos T., Yip C.K. (1993). "Optimal population size and endogenous growth", Economics Letters, 41, 107-110.

Pazner, E., Schmeidler, D. (1978). "Egalitarian equivalent allocations: A new concept of economic equity", Quarterly Journal of Economics, 92: 671-687.

Samuelson, P.A., Swamy, S. (1974). "Invariant economic index numbers and canonical duality: Survey and synthesis", American Economic Review, 64, 566-593. 
Scovronick, N., Budolfson, M., Dennig, F., Fleurbaey, M., Siebert, A., Socolow, R., Spears, D., Wagner, F. (2017). "Impacts of population growth and population ethics on optimal mitigation effort", PNAS, 114, 12 338-12343.

Weitzman, M.L. (2009). "On modeling and interpreting the economics of catastrophic climate change", Review of Economics and Statistics, 91, 1-19.

Zoli, C. (2009). "Variable population welfare and poverty orderings satisfying replication properties ", Working Paper Series - Department of Economics - University of Verona \# WP69, ISSN: 2036-4679.

\section{A Appendix: Proofs of the results}

\section{A.1 Proof of Proposition 1}

Let $R_{0} \in \mathcal{R}$ be a strictly monotonic preference ordering 8 satisfying the following property: there exist $\omega_{0} \in \mathcal{X}$ and $\varepsilon \in \mathbb{R}_{++}$such that for all $\lambda \in \mathbb{R}_{+}$and all $x \in \mathcal{X}$, if $x I_{0}\left((\lambda+\varepsilon) \omega_{0}\right)$ then $x \gg \lambda \omega_{0}$.

Consider any $i \in \mathbb{N}$. For every $\left(x_{i}, R_{i}\right) \in \mathcal{X} \times \mathcal{R}$, there is $\lambda \in \mathbb{R}_{++}$such that $x I_{i}\left(\lambda \omega_{0}\right)$. Hence $U\left((\lambda+\varepsilon) \omega_{0}, R_{0}\right) \subsetneq U\left(x_{i}, R_{i}\right),{ }^{9}$ and by Preference monotonicity $\left((\lambda+\varepsilon) \omega_{0}, R_{o}\right) \succsim\left(x_{i}, R_{i}\right)$. By strict monotonicity of $R_{0}, U\left(0, R_{0}\right)=\mathcal{X}$, and therefore $U\left(x_{i}, R_{i}\right) \subsetneq U\left(0, R_{0}\right)$ and $\left(x_{i}, R_{i}\right) \succsim\left(0, R_{0}\right)$. By continuity and increasingness of $R_{0}$, there is a unique $\mu \in \mathbb{R}_{+}$such that $\left(\mu \omega_{0}, R_{0}\right) \sim\left(x_{i}, R_{i}\right)$. By Anonymity, for any $j \in \mathbb{N} \backslash\{i\}$, any $\left(x_{j}, R_{j}\right) \in \mathbb{R}_{++}^{k} \times \mathcal{R}$ such that $x_{j}=x_{i}$ and $R_{j}=R_{i}$, we also have $\left(\mu \omega_{0}, R_{0}\right) \sim\left(x_{j}, R_{j}\right)$.

Define the set function $\psi: \mathcal{U} \rightarrow \mathbb{R}_{+}$as follows. For all $(x, R) \in \mathcal{X} \times \mathcal{R}$, $\psi(U(x, R))=\mu$ if $\left(\mu \omega_{0}, R_{0}\right) \sim(x, R)$. This is well-defined as $\mu$ is uniquely defined for any $(x, R)$ and by Lemma 1 , for any $R, R^{\prime} \in \mathcal{R}$ and any $x \in \mathcal{X}$, if $U(x, R)=U\left(x, R^{\prime}\right)$ then $(x, R) \sim\left(x, R^{\prime}\right)$. Set function $\psi$ is a continuous and increasing set function and we can define the associated well-being function $u_{\psi}: \mathcal{X} \times \mathcal{R} \rightarrow \mathbb{R}_{+}$. By definition, for any $i, j \in \mathbb{N}$, any $\left(x_{i}, R_{i}\right),\left(x_{j}, R_{j}\right) \in \mathcal{X} \times \mathcal{R}$,

\footnotetext{
8 "Strictly monotonic" means that if $x \geq x^{\prime}$ and $x \neq x^{\prime}$ then $x P_{0} x^{\prime}$.

${ }^{9}$ Indeed, for all $x^{\prime} \in U\left((\lambda+\varepsilon) \omega_{0}, R_{0}\right)$, we have $x^{\prime} \gg \lambda \omega_{0}$.
} 
$\left(x_{i}, R_{i}\right) \sim\left(\mu_{i} \omega_{0}, R_{0}\right)$ and $\left(x_{j}, R_{j}\right) \sim\left(\mu_{j} \omega_{0}, R_{0}\right)$ where $\mu_{i}=u_{\psi}\left(x_{i}, R_{i}\right)$ and $\mu_{j}=$ $u_{\psi}\left(x_{j}, R_{j}\right)$. So, by transitivity of $\succsim$ and monotonicity of $R_{0},\left(x_{i}, R_{i}\right) \succsim\left(x_{j}, R_{j}\right)$ if and only if $u_{\psi}\left(x_{i}, R_{i}\right) \geq u_{\psi}\left(x_{j}, R_{j}\right)$.

Consider any $n \in \mathbb{N}$ and let $N_{n}=\{1, \cdots, n\}$ and $\bar{R}_{n}=\left(R_{0}, \cdots, R_{0}\right)$. Let $\succsim_{n}^{\star}$ be a binary relation defined on $\mathbb{R}_{+}^{n}$ as follows: for all $z, z^{\prime} \in \mathbb{R}_{+}^{n} z \succsim_{n}^{\star} z^{\prime}$ if and only if there is $x_{N_{n}}, x_{N_{n}}^{\prime} \in \mathcal{X}^{N_{n}}$ such that $z_{i}=u_{\psi}\left(x_{i}, R_{0}\right)$ and $z_{i}^{\prime}=u_{\psi}\left(x_{i}^{\prime}, R_{0}\right)$ all $i \in N_{n}$, and $\left(x_{N_{n}}, \bar{R}_{n}\right) \succsim\left(x_{N_{n}}^{\prime}, \bar{R}_{n}\right)$. This relation is well defined: if $\hat{x}_{N_{n}}, \hat{x}_{N_{n}}^{\prime} \in \mathcal{X}^{N_{n}}$ are such that $z_{i}=u_{\psi}\left(\hat{x}_{i}, R_{0}\right)$ and $z_{i}^{\prime}=u_{\psi}\left(\hat{x}_{i}^{\prime}, R_{0}\right)$ all $i \in N_{n}$, then $\left(x_{N_{n}}, \bar{R}_{n}\right) \sim$ $\left(\hat{x}_{N_{n}}, \bar{R}_{n}\right)$ and $\left(x_{N_{n}}^{\prime}, \bar{R}_{n}\right) \sim\left(\hat{x}_{N_{n}}^{\prime}, \bar{R}_{n}\right)$. By definition, relation $\succsim_{n}^{\star}$ is transitive, complete and continuous. Hence there exists a continuous function $W_{n}: \mathbb{R}_{+}^{n} \rightarrow \mathbb{R}$ such that, for all $z, z^{\prime} \in \mathbb{R}_{+}^{n}$ :

$$
z \succsim_{n}^{\star} z^{\prime} \Longleftrightarrow W_{n}(z) \geq W_{n}\left(z^{\prime}\right)
$$

Without loss of generality, we can assume that $W_{n}$ is normalized. ${ }^{10}$

Betterness dominance and Anonymity imply that $W_{n}$ is monotonic and symmetric. And by definition, for all $x_{N_{n}}, x_{N_{n}}^{\prime} \in \mathcal{X}^{N_{n}}$ :

$$
\left(x_{N_{n}}, \bar{R}_{n}\right) \succsim\left(x_{N_{n}}^{\prime}, \bar{R}_{n}\right) \Longleftrightarrow W_{n}\left(\left(u_{\psi}\left(x_{i}, R_{0}\right)\right)_{i \in N_{n}}\right) \geq W_{n}\left(\left(u_{\psi}\left(x_{i}^{\prime}, R_{0}\right)\right)_{i \in N_{n}}\right)
$$

Consider any $N, M \in \mathcal{N}$ with $|N|=|M|=n$, all $R_{N} \in \mathcal{R}^{N}, R_{M}^{\prime} \in \mathcal{R}^{M}$, $x_{N} \in \mathcal{X}^{N}$ and $x_{M}^{\prime} \in \mathcal{X}^{M}$. Let $\sigma: N \rightarrow N_{n}$ and $\hat{\sigma}: M \rightarrow N_{n}$ be two bijections. By the results above, we know that for each $i \in N,\left(x_{i}, R_{i}\right) \sim\left(u_{\psi}\left(x_{i}, R_{i}\right) \omega_{0}, R_{0}\right)$, and for each $j \in M,\left(x_{j}^{\prime}, R_{j}^{\prime}\right) \sim\left(u_{\psi}\left(x_{j}^{\prime}, R_{j}^{\prime}\right) \omega_{0}, R_{0}\right)$. Let $\hat{x}_{N_{n}}, \hat{x}_{N_{n}}^{\prime} \in \mathcal{X}^{N_{n}}$ be defined as $\hat{x}_{\sigma(i)}=u_{\psi}\left(x_{i}, R_{i}\right) \omega_{0}$ for all $i \in N$ and $\hat{x}_{\hat{\sigma}(j)}^{\prime}=u_{\psi}\left(x_{j}^{\prime}, R_{j}^{\prime}\right) \omega_{0}$ for all $j \in M$, so that $u_{\psi}\left(\hat{x}_{\sigma(i)}, R_{0}\right)=u_{\psi}\left(x_{i}, R_{i}\right)$ for all $i \in N$ and $u_{\psi}\left(\hat{x}_{\hat{\sigma}(j)}^{\prime}, R_{0}\right)=u_{\psi}\left(x_{j}^{\prime}, R_{j}^{\prime}\right)$ for all $j \in M$. Therefore, by Anonymity, Continuity and Betterness dominance, $\left(x_{N}, R_{N}\right) \sim\left(\hat{x}_{N_{n}}, \bar{R}_{n}\right)$ and $\left(x_{M}^{\prime}, R_{M}^{\prime}\right) \sim\left(\hat{x}_{N_{n}}^{\prime}, \bar{R}_{n}\right)$.

\footnotetext{
${ }^{10}$ Indeed, $W_{n}$ is an ordinal representation of $\succsim_{n}^{\star}$ that can be transformed by an increasing function so that it is normalized.
} 
Gathering all our results, we obtain the following equivalence:

$$
\begin{aligned}
\left(x_{N}, R_{N}\right) \succsim\left(x_{M}^{\prime}, R_{M}^{\prime}\right) & \Longleftrightarrow\left(\hat{x}_{N_{n}}, \bar{R}_{n}\right) \succsim\left(\hat{x}_{N_{n}}^{\prime}, \bar{R}_{n}\right) \\
& \Longleftrightarrow W_{n}\left(\left(u_{\psi}\left(\hat{x}_{k}, R_{0}\right)\right)_{k \in N_{n}}\right) \geq W_{n}\left(\left(u_{\psi}\left(\hat{x}_{k}^{\prime}, R_{0}\right)\right)_{k \in N_{n}}\right) \\
& \Longleftrightarrow W_{n}\left(\left(u_{\psi}\left(x_{i}, R_{i}\right)\right)_{i \in N}\right) \geq W_{n}\left(\left(u_{\psi}\left(x_{j}^{\prime}, R_{j}^{\prime}\right)\right)_{j \in M}\right),
\end{aligned}
$$

where the last equivalence is due to the symmetry of $W_{n}$.

\section{A.2 Preliminary results for Theorems 2 and 3}

Let us now present preliminary results on social orderings over acts in $\overline{\mathcal{F}}$. These results are key steps in the proof of Theorems 2 and 3.

Proposition A.1 If a fair universal social welfare ordering $\succsim$ satisfies Indifferent addition and Restricted social expected utility, then there exists a continuous and increasing set function $\psi$, a function $V: \mathbb{N} \times \mathbb{R}_{+}$that is continuous and increasing in its second argument, and for each $n \in \mathbb{N}$ a continuous, symmetric, monotonic, and normalized function $W_{n}: \mathbb{R}_{+}^{n} \rightarrow \mathbb{R}$ such that, for all $f, g \in \overline{\mathcal{F}}$ :

$$
\begin{aligned}
f \succsim g \Longleftrightarrow & \mathbb{E}\left[V\left(n(f), W_{n(f)}\left(\left(u_{\psi}\left(x_{i}(f), R_{i}(f)\right)\right)_{i \in N(f)}\right)\right)\right] \\
& \geq \mathbb{E}\left[V\left(n(g), W_{n(g)}\left(\left(u_{\psi}\left(x_{i}(g)\right), R_{i}(g)\right)_{i \in N(g)}\right)\right)\right]
\end{aligned}
$$

Proof. By Prop. 1, given that the social welfare ordering $\succsim$ on $\mathcal{F}$ is a fair universal social welfare ordering then there exists a continuous, increasing set function $\psi: \mathcal{U} \rightarrow \mathbb{R}$ and for each $n \in \mathbb{N}$ a continuous, symmetric, monotonic, and normalized function $W_{n}: \mathbb{R}_{+}^{n} \rightarrow \mathbb{R}$ such that, for all $N, M \in \mathcal{N}$ with $|N|=$ $|M|=n$, all $R_{N} \in \mathcal{R}^{N}, R_{M}^{\prime} \in \mathcal{R}^{M}, x_{N} \in \mathcal{X}^{N}$ and $x_{M}^{\prime} \in \mathcal{X}^{M}$ :

$$
\left(x_{N}, R_{N}\right) \succsim\left(x_{M}^{\prime}, R_{M}^{\prime}\right) \Longleftrightarrow W_{n}\left(\left(u_{\psi}\left(x_{i}, R_{i}\right)\right)_{i \in N}\right) \geq W_{n}\left(\left(u_{\psi}\left(x_{j}^{\prime}, R_{j}^{\prime}\right)\right)_{j \in M}\right)
$$

Let ${ }^{\star}$ be a binary relation defined on $\mathcal{Z}=\cup_{n \in \mathbb{N}} \mathbb{R}_{+}^{n}$ as follows: for all $z, z^{\prime} \in \mathcal{Z}$ 
(with $z \in \mathbb{R}_{+}^{n}$ and $z^{\prime} \in \mathbb{R}_{+}^{m}$ ), $z \succsim^{\star} z^{\prime}$ if and only if there exist $N, M \in \mathcal{N}$ with $|N|=n$ and $|M|=m, x_{N} \in \mathcal{X}^{N}, R_{N} \in \mathcal{R}^{N}, x_{M}^{\prime} \in \mathcal{X}^{M}$ and $R_{M}^{\prime} \in \mathcal{R}^{M}$, and bijections $\sigma: N \rightarrow N_{n}$ and $\hat{\sigma}: M \rightarrow N_{m}$, such that $z_{\sigma(i)}=u_{\psi}\left(x_{i}, R_{i}\right)$ for all $i \in N$, and $z_{\hat{\sigma}(j)}^{\prime}=u_{\psi}\left(x_{j}^{\prime}, R_{j}^{\prime}\right)$ for all $j \in M$, and $\left(x_{N}, R_{N}\right) \succsim\left(x_{M}^{\prime}, R_{M}^{\prime}\right)$. As in the proof of Prop. 1, this relation is well defined and, for all $n \in \mathbb{N}, z, z^{\prime} \in \mathbb{R}_{+}^{n}$ :

$$
z \succsim^{\star} z^{\prime} \Longleftrightarrow W_{n}(z) \geq W_{n}\left(z^{\prime}\right)
$$

Furthermore, because $\succsim$ satisfies Indifferent addition, $\succsim^{\star}$ satisfies the following condition: for all $n \in \mathbb{N}, z \in \mathbb{R}_{+}^{n}$, there exists $t \in \mathbb{R}_{+}$such that $z \sim^{\star} z^{\prime}$, where $z^{\prime} \in \mathbb{R}_{+}^{n+1}$ is such that $z_{i}^{\prime}=z_{i}$ for all $i \in N_{n}$ and $z_{n+1}^{\prime}=t$. Therefore, by repeated application of this property, for any $n \in \mathbb{N}, m \in \mathbb{N}$ and $z \in \mathbb{R}_{+}^{n}$, there exists $z^{\prime} \in \mathbb{R}_{+}^{n+m}$ such that $z \sim^{\star} z^{\prime}$. By continuity of $W_{n+m}$, the sets $\left\{z^{\prime \prime} \in \mathbb{R}_{+}^{n+m} \mid z^{\prime \prime} \succsim^{\star} z^{\prime}\right\}$ and $\left\{z^{\prime \prime} \in \mathbb{R}_{+}^{n+m} \mid z^{\prime} \succsim^{\star} z^{\prime \prime}\right\}$ are closed. By transitivity of $\succsim^{\star},\left\{z^{\prime \prime} \in \mathbb{R}_{+}^{n+m} \mid z^{\prime \prime} \succsim^{\star} z^{\prime}\right\}=\left\{z^{\prime \prime} \in \mathbb{R}_{+}^{n+m} \mid z^{\prime \prime} \succsim^{\star} z\right\}$ and $\left\{z^{\prime \prime} \in \mathbb{R}_{+}^{n+m} \mid z^{\prime} \succsim^{*}\right.$ $\left.z^{\prime \prime}\right\}=\left\{z^{\prime \prime} \in \mathbb{R}_{+}^{n+m} \mid z \succsim^{\star} z^{\prime \prime}\right\}$. Hence, for any $n \in \mathbb{N}, m \in \mathbb{N}$ and $z \in \mathbb{R}_{+}^{n}$, the sets $\left\{z^{\prime \prime} \in \mathbb{R}_{+}^{n+m} \mid z^{\prime \prime} \succsim^{\star} z\right\}$ and $\left\{z^{\prime \prime} \in \mathbb{R}_{+}^{n+m} \mid z \succsim^{\star} z^{\prime \prime}\right\}$ are closed. This is the Extended continuity property of Blackorby, Bossert and Donaldson (2001). By Theorem 1 in Blackorby, Bossert and Donaldson (2001), there exists a value function $\bar{\Upsilon}: \mathbb{N} \times \mathbb{R}_{+} \rightarrow \mathbb{R}$, continuous and increasing in its second argument, such that, for all $n, m \in \mathbb{N}$, for all $u \in \mathbb{R}_{+}^{n}$, and for all $v \in \mathbb{R}_{+}^{m}$,

$$
u \succsim^{\star} v \Longleftrightarrow \bar{\Upsilon}\left(n, W_{n}(u)\right) \geq \bar{\Upsilon}\left(m, W_{m}(v)\right)
$$

By the previous steps, this implies that for all $N, M \in \mathcal{N}$, all $R_{N} \in \mathcal{R}^{N}$ and $R_{M}^{\prime} \in \mathcal{R}^{M}$, and all $x_{N} \in \mathcal{X}^{N}$ and $x_{M}^{\prime} \in \mathcal{X}^{M}$ :

$\left(x_{N}, R_{N}\right) \succsim\left(x_{M}^{\prime}, R_{M}^{\prime}\right) \Longleftrightarrow \bar{\Upsilon}\left(|N|, W_{|N|}\left(\left(u_{\psi}\left(x_{i}, R_{i}\right)\right)_{i \in N}\right)\right) \geq \bar{\Upsilon}\left(|M|, W_{|M|}\left(\left(u_{\psi}\left(x_{j}^{\prime}, R_{j}^{\prime}\right)\right)_{j \in M}\right)\right)$

But, by Restricted social expected utility, there exists a continuous function 
$\tilde{V}: \mathcal{A} \rightarrow \mathbb{R}$ such that, for all $f, g \in \overline{\mathcal{F}}:$

$$
f \succsim g \Longleftrightarrow \mathbb{E}[\tilde{V}(f)] \geq \mathbb{E}[\tilde{V}(g)] .
$$

In particular, this implies that for all $N, M \in \mathcal{N}$, all $R_{N} \in \mathcal{R}^{N}$ and $R_{M}^{\prime} \in \mathcal{R}^{M}$, and all $x_{N} \in \mathcal{X}^{N}$ and $x_{M}^{\prime} \in \mathcal{X}^{M}$ :

$$
\left(x_{N}, R_{N}\right) \succsim\left(x_{M}^{\prime}, R_{M}^{\prime}\right) \Longleftrightarrow \tilde{V}\left(x_{N}, R_{N}\right) \geq \tilde{V}\left(x_{M}^{\prime}, R_{M}^{\prime}\right)
$$

Therefore, there must exists a continuous and increasing function $\Theta: \mathbb{R} \rightarrow \mathbb{R}$ such that, for all $N \in \mathcal{N}, R_{N} \in \mathcal{R}^{N}$ and $x_{N} \in \mathcal{X}^{N}$ :

$$
\tilde{V}\left(x_{N}, R_{n}\right)=\Theta \circ \bar{\Upsilon}\left(|N|, W_{|N|}\left(\left(u_{\psi}\left(x_{i}, R_{i}\right)\right)_{i \in N}\right)\right) .
$$

Denoting $V=\Theta \circ \bar{\Upsilon}$, we obtain the result.

Proposition A.2 If a fair universal social welfare ordering $\succsim$ satisfies Indifferent addition, Extended replication invariance and Restricted social expected utility then there exists a continuous and increasing set function $\psi$, a continuous, symmetric, monotonic and replication invariant function $\Gamma: \mathcal{Z} \rightarrow \mathbb{R}, \alpha \in \mathbb{R}$ and $\beta \in \mathbb{R}$ such that, for all $f, g \in \overline{\mathcal{F}}$ :

1. If $\alpha \neq 0$,

$$
\begin{aligned}
f \succsim g \Longleftrightarrow \mathbb{E} & {\left[(n(f))^{\alpha}\left(\Gamma\left(\left(u_{\psi}\left(x_{i}(f), R_{i}(f)\right)\right)_{i \in N(f)}\right)-\beta\right)\right] \geq } \\
& \mathbb{E}\left[(n(g))^{\alpha}\left(\Gamma\left(\left(u_{\psi}\left(x_{i}(g), R_{i}(g)\right)\right)_{i \in N(g)}\right)-\beta\right)\right]
\end{aligned}
$$

2. If $\alpha=0$,

$$
\begin{aligned}
f \succsim g \Longleftrightarrow \mathbb{E} & {\left[\Gamma\left(\left(u_{\psi}\left(x_{i}(f), R_{i}(f)\right)\right)_{i \in N(f)}\right)-\beta \ln (n(f))\right] \geq } \\
& \mathbb{E}\left[\Gamma\left(\left(u_{\psi}\left(x_{i}(g), R_{i}(g)\right)\right)_{i \in N(g)}\right)-\beta \ln (n(g))\right]
\end{aligned}
$$


Proof. By Proposition A.1, given that $\succsim$ is a fair universal social welfare ordering that satisfies Indifferent addition and Restricted social expected utility, there exists a continuous and increasing set function $\psi$, a function $V: \mathbb{N} \times \mathbb{R}_{+}$, continuous and increasing in its second argument, and for each $n \in \mathbb{N}$ a continuous, symmetric, monotonic, and normalized function $W_{n}: \mathbb{R}_{+}^{n} \rightarrow \mathbb{R}$ such that, for all $f, g \in \overline{\mathcal{F}}:$

$$
\begin{aligned}
f \succsim g \Longleftrightarrow & \mathbb{E}\left[V\left(n(f), W_{n(f)}\left(\left(u_{\psi}\left(x_{i}(f), R_{i}(f)\right)\right)_{i \in N(f)}\right)\right)\right] \\
& \geq \mathbb{E}\left[V\left(n(g), W_{n(g)}\left(\left(u_{\psi}\left(x_{i}(g), R_{i}(g)\right)\right)_{i \in N(g)}\right)\right)\right]
\end{aligned}
$$

Step 1: For $n \in \mathbb{N}$, let $\phi_{n}$ be the function such that $\phi_{n}(t)=V(n, t)$ for all $t \in \mathbb{R}_{+}$. We prove that for all $n, m \in \mathbb{N}$ and $t \in \mathbb{R}_{+}, \phi_{n m}(t)=\alpha_{n} \phi_{m}(t)+\beta_{n, m}$ for some parameters $\alpha_{n}, \beta_{n, m}$.

For $m, m^{\prime} \in \mathbb{N}$, define $\succsim_{m, m^{\prime}}$, a relation on $\mathbb{R}_{+}^{2}$, as follows: for all $(t, v),\left(t^{\prime}, v^{\prime}\right) \in$ $\mathbb{R}_{+}^{2},(t, v) \succsim_{m, m^{\prime}}\left(t^{\prime}, v^{\prime}\right)$ if there exist $f, g \in \overline{\mathcal{F}}$ such that $f \succsim g$ and $f, g$ are defined as follows:

- there exists $E \in \mathcal{E}$ with $p(E)=\pi>0$;

- there exist $M, M^{\prime} \in \mathcal{N}$ with $M \cap M^{\prime}=\emptyset,|M|=m$ and $\left|M^{\prime}\right|=m^{\prime}$;

- there exist $\left(x_{M}, R_{M}\right),\left(\hat{x}_{M^{\prime}}, \hat{R}_{M^{\prime}}\right),\left(x_{M}^{\prime}, R_{M}^{\prime}\right),\left(\hat{x}_{M^{\prime}}^{\prime}, \hat{R}_{M^{\prime}}^{\prime}\right) \in \mathcal{A}$, such that $t=u_{\psi}\left(x_{i}, R_{i}\right)$ and $t^{\prime}=u_{\psi}\left(x_{i}^{\prime}, R_{i}^{\prime}\right)$ for all $i \in M, v=u_{\psi}\left(\hat{x}_{j}, \hat{R}_{j}\right)$, and $v^{\prime}=u_{\psi}\left(\hat{x}_{j}^{\prime}, \hat{R}_{j}^{\prime}\right)$ for all $j \in M^{\prime}$

and $f(s)=\left(x_{M}, R_{M}\right), g(s)=\left(x_{M}^{\prime}, R_{M}^{\prime}\right)$ for all $s \in E$, while $f(s)=\left(\hat{x}_{M^{\prime}}, \hat{R}_{M^{\prime}}\right)$ and $g(s)=\left(\hat{x}_{M^{\prime}}^{\prime}, \hat{R}_{M^{\prime}}^{\prime}\right)$ for all $s \in \mathcal{S} \backslash E$.

By definition, for any $m, m^{\prime} \in \mathbb{N}$ and $(t, v),\left(t^{\prime}, v^{\prime}\right) \in \mathbb{R}_{+}^{2}$ :

$$
(t, v) \succsim_{m, m^{\prime}}\left(t^{\prime}, v^{\prime}\right) \Longleftrightarrow \pi \phi_{m}(t)+(1-\pi) \phi_{m^{\prime}}(v) \geq \pi \phi_{m}\left(t^{\prime}\right)+(1-\pi) \phi_{m^{\prime}}\left(v^{\prime}\right)
$$


But by extended replication invariance and by definition, for all $n \in \mathbb{N}$ :

$$
\begin{aligned}
(t, v) \succsim_{m, m^{\prime}}\left(t^{\prime}, v^{\prime}\right) & \Longleftrightarrow(t, v) \succsim_{n m, n m^{\prime}}\left(t^{\prime}, v^{\prime}\right) \\
& \Longleftrightarrow \pi \phi_{n m}(t)+(1-\pi) \phi_{n m^{\prime}}(v) \geq \pi \phi_{n m}\left(t^{\prime}\right)+(1-\pi) \phi_{n m^{\prime}}\left(v^{\prime}\right) .
\end{aligned}
$$

Hence, there exists an increasing function $\Psi_{n, m, m^{\prime}}: \mathbb{R} \rightarrow \mathbb{R}$ such that, for any $(t, v) \in \mathbb{R}_{+}^{2}:$

$$
\begin{aligned}
\Psi_{n, m, m^{\prime}}\left(\pi \phi_{m}(t)+(1-\pi) \phi_{m^{\prime}}(v)\right) & =\pi \phi_{n m}(t)+(1-\pi) \phi_{n m^{\prime}}(v) \\
& =\pi \phi_{n m} \circ \phi_{m}^{-1}\left(\frac{\pi}{\pi} \phi_{m}(t)\right)+(1-\pi) \phi_{n m^{\prime}} \circ \phi_{m^{\prime}}^{-1}\left(\frac{1-\pi}{1-\pi} \phi_{m^{\prime}}(v)\right)
\end{aligned}
$$

Define the sets $T=\pi \phi_{m}\left(\mathbb{R}_{+}\right)$and $Y=(1-\pi) \phi_{m^{\prime}}\left(\mathbb{R}_{+}\right)$, and the functions $\Phi_{n, m}(t)=\phi_{n m} \circ \phi_{m}^{-1}\left(\frac{t}{\pi}\right)$ for all $t \in T$, and $\Phi_{n, m^{\prime}}(y)=\phi_{n m^{\prime}} \circ \phi_{m^{\prime}}^{-1}\left(\frac{y}{1-\pi}\right)$ for all $y \in Y$. Given that $\phi_{n}$ is continuous and increasing for all $n \in N, T, Y$ are intervals in $\mathbb{R}$, and $\Phi_{n m}$ and $\Phi_{n m^{\prime}}$ are continuous and increasing functions. We obtain the following functional equation: For all $(t, y) \in T \times Y$

$$
\Psi_{n, m, m^{\prime}}(t+y)=\Phi_{n, m}(t)+\Phi_{n, m^{\prime}}(y)
$$

This is a generalized Pexider functional equation, and it is known that in that case $\Psi_{n, m, m^{\prime}}, \Phi_{n, m}$ and $\Phi_{n, m^{\prime}}$ must be affine (Radó and Baker, 1987). Hence there exist $\alpha_{n, m, m^{\prime}} \in \mathbb{R}_{++}, \beta_{n, m, m^{\prime}} \in \mathbb{R}$ and $\beta_{n, m, m^{\prime}}^{\prime} \in \mathbb{R}$ such that $\phi_{n m}(t)=$ $\alpha_{n, m, m^{\prime}} \phi_{m}(t)+\beta_{n, m, m^{\prime}}$ and $\phi_{n m^{\prime}}(y)=\alpha_{n, m, m^{\prime}} \phi_{m^{\prime}}(y)+\beta_{n, m, m^{\prime}}^{\prime}$.

Given that this true for any $m, m^{\prime} \in \mathbb{N}$, we obtain that

$$
\phi_{n m}(t)=\alpha_{n, m, m^{\prime}} \phi_{n}(t)+\beta_{n, m, m^{\prime}}=\alpha_{n, m, m^{\prime \prime}} \phi_{n}(t)+\beta_{n, m, m^{\prime \prime}}
$$

for all $m^{\prime}, m^{\prime \prime}$ so that $\alpha_{n, m, m^{\prime}}=\bar{\alpha}_{n, m}$ and $\beta_{n, m, m^{\prime}}=\bar{\beta}_{n, m}$ for some $\bar{\alpha}_{n, m}, \bar{\beta}_{n, m}$ and for all $m^{\prime} \in \mathbb{N}$. Similarly,

$$
\phi_{n m^{\prime}}(y)=\bar{\alpha}_{n, m} \phi_{m^{\prime}}(y)+\beta_{n, m, m^{\prime}}^{\prime}=\bar{\alpha}_{n, \hat{m}} \phi_{m^{\prime}}(y)+\beta_{n, \hat{m}, m^{\prime}}^{\prime}
$$


for all $m, \hat{m}$ so that $\bar{\alpha}_{n, m}=\alpha_{n}$ and $\beta_{n, m, m^{\prime}}^{\prime}=\bar{\beta}_{n, m^{\prime}}^{\prime}$ for some $\alpha_{n}, \bar{\beta}_{n, m^{\prime}}^{\prime}$ and for all $m \in \mathbb{N}$. It is also the case that $\beta_{n, m, m^{\prime}}^{\prime}=\beta_{n, m^{\prime}, m}$, so that $\bar{\beta}_{n, m}=\bar{\beta}_{n, m}^{\prime}=\beta_{n, m}$ for some $\beta_{n, m}$ and for all $n, m \in \mathbb{N}$.

Step 2: Let $\succsim^{\star}$ be a binary relation defined on $\mathcal{Z}=\cup_{k \in \mathbb{N}} \mathbb{R}_{+}^{k}$ as in the proof of Proposition A.1. We characterize its representation.

We already know by Proposition A.1 that, for any $z, z^{\prime} \in \mathcal{Z} z \succsim^{\star} z^{\prime}$ if and only if $W(z) \geq W\left(z^{\prime}\right)$, where $W(z)=V\left(n, W_{n}(z)\right)$ for all $z \in \mathbb{R}_{+}^{n}$. By the previous step, we know that for some family of functions $\phi_{m}$ and some parameters $\alpha_{n}, \beta_{n, m}$, for all $n, m \in \mathbb{N}$ and $t \in \mathbb{R}_{+} V(n m, t)=\phi_{n m}(t)=\alpha_{n} \phi_{m}(t)+\beta_{n, m}$.

Consider any $t \in \mathbb{R}_{+}$and any $m, m^{\prime} \in \mathbb{N}$ with $m^{\prime} \geq m$ (without loss of generality). By continuity and monotonicity of $W_{n}$ and using indifferent addition, we know that there exists $v \in \mathbb{R}_{+}$such that $\phi_{m}(t)=\phi_{m^{\prime}}(v)$. But by replication invariance, we must also have $\phi_{n m}(t)=\phi_{n m^{\prime}}(v)$. This means that $\alpha_{n} \phi_{m}(t)+$ $\beta_{n, m}=\alpha_{n} \phi_{m^{\prime}}(v)+\beta_{n, m^{\prime}}$ and therefore $\beta_{n, m}=\beta_{n, m^{\prime}}$. This is true for all $m, m^{\prime} \in \mathbb{N}$ so that $\beta_{n, m}=\beta_{n}$ for some $\beta_{n}$ and for all $m \in \mathbb{N}$.

Consider any $m \in \mathbb{N}$ and $z \in \mathbb{R}_{+}^{m}$. Let $t=W_{m}(z)$. By normalization of $W_{m}$, we know that $z \sim^{\star} t \cdot 1_{m}$ and that $W_{m}\left(t \cdot 1_{m}\right)=W_{m^{\prime}}\left(t \cdot 1_{m^{\prime}}\right)$ for all $m^{\prime} \neq m$. Hence, if $z^{\prime} \in \mathcal{Z}$ is a $n$-replica of $z$, we have, by replication invariance $W_{m}(z)=$ $W_{m}\left(t \cdot 1_{m}\right)=W_{n m}\left(t \cdot 1_{n m}\right)=W_{n m}\left(z^{\prime}\right)$.

So, to summarize, there exist sequences $\left(\alpha_{n}\right)_{n \in \mathbb{N}}$ and $\left(\beta_{n}\right)_{n \in \mathbb{N}}$ such that for all $n \in \mathbb{N}$, for all $z, z^{\prime} \in \mathcal{Z}$ such that $z \in \mathbb{R}_{+}^{m}$ and $z^{\prime}$ is a $n$-replica of $z$, we have: $W\left(z^{\prime}\right)=\alpha_{n} \phi_{m}\left(W_{n m}\left(z^{\prime}\right)\right)+\beta_{n}=\alpha_{n} \phi_{m}\left(W_{m}(z)\right)+\beta_{n}=\alpha_{n} V\left(m, W_{m}(z)\right)+$ $\beta_{n}=\alpha_{n} W(z)+\beta_{n}$. This is the property named 'Generalized Affine Population Replication' by Zoli (2009).

By proposition 3.1 in Zoli (2009), we thus know there exist constants $\alpha, \beta \in \mathbb{R}$, and an increasing continuous replication-invariant function $\Gamma: \mathbb{R}_{+} \rightarrow \mathbb{R}$ such that for all $n \in \mathbb{N}$ and $z \in \mathbb{R}_{+}^{n}$ :

$$
W(z)=\left\{\begin{array}{l}
n^{\alpha}(\Gamma(z)-\beta) \text { if } \alpha \neq 0 \\
\Gamma(z)+\beta \ln (n) \text { if } \alpha=0
\end{array}\right.
$$


By definition, then, in Eq. (A.1), for any $f \in \overline{\mathcal{F}}$ for all $s \in \mathcal{S}$,

$V\left(n(f(s)), W_{n(f(s))}\left(\left(u_{\psi}\left(x_{i}(f(s)), R_{i}(f(s))\right)\right)_{i \in N(f(s))}\right)=W\left(\left(u_{\psi}\left(x_{i}(f(s)), R_{i}(f(s))\right)\right)_{i \in N(f(s))}\right)\right.$.

Proposition A.3 If a fair universal social welfare ordering $\succsim$ satisfies Indifferent addition, Independence of the sure and Restricted social expected utility then there exists a continuous and increasing set function $\psi$, a constant $\alpha \in \mathbb{R}, a$ function $\varphi: \mathbb{R}_{+} \rightarrow \mathbb{R}$, and for each $n \in \mathbb{N}$ a constant $c_{n} \in D_{\varphi}^{n}$ such that:

1. Either $\alpha \neq 0$ and for all $f, g \in \bar{F}$ :

$$
f \succsim g \Longleftrightarrow \frac{1}{\alpha} \mathbb{E}\left[e^{\alpha\left[\sum_{i \in N(f)} \varphi\left(u_{\psi}\left(x_{i}(f), R_{i}(f)\right)\right)-c_{n(f)}\right]}\right] \geq \frac{1}{\alpha} \mathbb{E}\left[e^{\alpha\left[\sum_{i \in N(g)} \varphi\left(u_{\psi}\left(x_{i}(g), R_{i}(g)\right)\right)-c_{n(g)}\right]}\right]
$$

2. Or for all $f, g \in \bar{F}$ :

$$
f \succsim g \Longleftrightarrow \mathbb{E}\left[\sum_{i \in N(f)} \varphi\left(u_{\psi}\left(x_{i}(f), R_{i}(f)\right)\right)-c_{n(f)}\right] \geq \mathbb{E}\left[\sum_{i \in N(g)} \varphi\left(u_{\psi}\left(x_{i}(g), R_{i}(g)\right)\right)-c_{n(g)}\right] .
$$

\section{Proof.}

Step 1: An additively separable function when there is no risk.

By Proposition A.1, given that $\succsim$ is a fair universal social welfare ordering that satisfies Indifferent addition and Restricted social expected utility, there exists a continuous and increasing set function $\psi$, a function $V: \mathbb{N} \times \mathbb{R}_{+}$, continuous and increasing in its second argument, and for each $n \in \mathbb{N}$ an increasing, continuous, monotonic and normalized function $W_{n}$ such that, for all $f, g \in \bar{F}$ :

$$
\begin{aligned}
f \succsim g \Longleftrightarrow & \mathbb{E}\left[V\left(n(f), W_{n(f)}\left(\left(u_{\psi}\left(x_{i}(f), R_{i}(f)\right)\right)_{i \in N(f)}\right)\right)\right] \\
& \geq \mathbb{E}\left[V\left(n(g), W_{n(g)}\left(\left(u_{\psi}\left(x_{i}(g), R_{i}(g)\right)\right)_{i \in N(g)}\right)\right)\right]
\end{aligned}
$$

Let define relation $\succsim^{\star}$ on $\mathcal{Z}$ like in the proof of Prop. A.1, so that for all 
$n, m \in \mathbb{N}$, for all $z \in \mathbb{R}_{+}^{n}$, and for all $z^{\prime} \in \mathbb{R}_{+}^{m}$,

$$
z \succsim^{\star} z^{\prime} \Longleftrightarrow V\left(n, W_{n}(z)\right) \geq V\left(m, W_{m}\left(z^{\prime}\right)\right)
$$

By definition (and by the properties of $W_{n}$ ), relation $\succsim^{\star}$ is continuous, symmetric and monotonic. Because $\succsim$ satisfies Indifferent addition and Independence of the sure, $\succsim^{\star}$ satisfies Intermediate existence of critical levels and utility independence in the sense of Blackorby, Bossert and Donaldson (2005). It also satisfies Strong Pareto in the sense of Blackorby, Bossert and Donaldson (2005). ${ }^{11}$ Therefore, by Theorem 6.5 in Blackorby, Bossert and Donaldson (2005), there exists a continuous and increasing function $\varphi: \mathbb{R}_{+} \rightarrow \mathbb{R}$ and for each $n \in \mathbb{N}$ a constant $\gamma_{n} \in \varphi\left(\mathbb{R}_{+}\right)$such that, for all $z, z^{\prime} \in \mathcal{Z}$ with $z \in \mathbb{R}_{+}^{n}$ and $z^{\prime} \in \mathbb{R}_{+}^{m}$,

$$
z R z^{\prime} \Longleftrightarrow \sum_{i=1}^{n} \varphi\left(z_{i}\right)-\sum_{i=1}^{n} \gamma_{i} \geq \sum_{i=1}^{m} \varphi\left(z_{i}^{\prime}\right)-\sum_{i=1}^{m} \gamma_{i}
$$

By definition of $\succsim^{\star}$, for any $N, M \in \mathcal{N},\left(x_{N}, R_{N}\right) \in(\mathcal{X} \times \mathcal{R})^{N},\left(x_{M}^{\prime}, R_{M}^{\prime}\right) \in$ $(\mathcal{X} \times \mathcal{R})^{M}$ :

$$
\left(x_{N}, R_{N}\right) \succsim\left(x_{M}^{\prime}, R_{M}^{\prime}\right) \Longleftrightarrow\left(u_{\psi}\left(x_{i}, R_{i}\right)\right)_{i \in N} \succsim^{\star}\left(u_{\psi}\left(x_{i}^{\prime}, R_{i}^{\prime}\right)\right)_{i \in M},
$$

and therefore, by our result above:

$\left(x_{N}, R_{N}\right) \succsim\left(x_{M}^{\prime}, R_{M}^{\prime}\right) \Longleftrightarrow \sum_{i \in N} \varphi \circ u_{\psi}\left(x_{i}, R_{i}\right)-\sum_{i=1}^{|N|} \gamma_{i} \geq \sum_{j \in M} \varphi \circ u_{\psi}\left(x_{j}^{\prime}, R_{j}^{\prime}\right)-\sum_{i=1}^{|M|} \gamma_{i}$.

\footnotetext{
${ }^{11}$ Indeed, consider any $z, z^{\prime} \in \mathbb{R}_{+}^{n}$ such that for some $i z_{i}>z_{i}^{\prime}$ and $z_{j}=z_{j}^{\prime}$ for all $j \in$ $\{1, \cdots, n\} \backslash\{i\}$ : let us prove that $z \succ z^{\prime}$. By monotonicity $(\underbrace{z_{i}, \cdots, z_{i}}_{n \text { times }}) \succ(\underbrace{z_{i}^{\prime}, \cdots, z_{i}^{\prime}}_{n \text { times }})$. There must exist $k \in\{1, \cdots, n\}$ such that, if we define $z^{k}, \tilde{z}^{k} \in \mathbb{R}_{+}^{n}$ by $z_{l}^{k}=\tilde{z}_{l}^{k}=z_{i}$ for all $l<k$, $z_{j}^{k}=\tilde{z}_{j}^{k}=z_{i}^{\prime}$ for all $j>k, z_{k}^{k}=z_{i}$ and $\tilde{z}_{k}^{k}=z_{i}^{\prime}$, we must have $z^{k} \succ \tilde{z}^{k}$ (otherwise by transitivity we would have $\left.(\underbrace{z_{i}, \cdots, z_{i}}_{n \text { times }}) \precsim(\underbrace{z_{i}^{\prime}, \cdots, z_{i}^{\prime}}_{n \text { times }})\right)$. Let us define $\hat{z}, \hat{z}^{\prime} \in \mathbb{R}_{+}^{n}$ such that $\hat{z}_{l}=z_{\pi(l)}^{k}$ and $\hat{z}_{l}^{\prime}=\tilde{z}_{\pi(l)}^{k}$ for $l \in\{1, \cdots, n\}$ where $\pi$ is a bijection such that $\pi(i)=k, \pi(k)=i$ and $\pi(l)=l$ for all $l \neq i, k$. By symmetry, because $z^{k} \succ \tilde{z}^{k}$, we obtain $\hat{z} \succ \hat{z}^{\prime}$. By utility independence, this implies $z \succ z^{\prime}$.
} 
Step 2. For any $\left(x_{N}, R_{N}\right) \in \mathcal{A}$, denote $\tilde{W}\left(x_{N}, R_{N}\right)=V\left(|N|, W_{|N|}\left(\left(u_{\psi}\left(x_{i}, R_{i}\right)\right)_{i \in N}\right)\right)$, where $V$ and $W_{|N|}$ are the function in Equation A.2. Equation A.2 implies that, for all $N, M \in \mathcal{N}$, all $R_{N} \in \mathcal{R}^{N}$ and $R_{M}^{\prime} \in \mathcal{R}^{M}$, and all $x_{N} \in(\mathcal{X})^{N}$ and $x_{M}^{\prime} \in(\mathcal{X})^{M}$ :

$$
\left(x_{N}, R_{N}\right) \succsim\left(x_{M}^{\prime}, R_{M}^{\prime}\right) \Longleftrightarrow \tilde{W}\left(x_{N}, R_{N}\right) \geq \tilde{W}\left(x_{M}^{\prime}, R_{M}^{\prime}\right)
$$

By Step 1, there must exist a a continuous and increasing function $\Phi$ such that, for any $N \in \mathcal{N}, R_{N} \in \mathcal{R}^{N}$ and $x_{N} \in \mathcal{X}^{N}$ :

$$
\tilde{W}\left(x_{N}, R_{N}\right)=\Phi\left(\sum_{i \in N} \varphi \circ u_{\psi}\left(x_{i}, R_{i}\right)-\sum_{i=1}^{|N|} \gamma_{i}\right)
$$

We normalize the representation so that $\gamma_{1}=\min \varphi\left(\mathbb{R}_{+}\right)$.

Given this representation, let $T=\{t \in \mathbb{R} \mid \exists(x, R) \in \mathcal{X} \times \mathcal{R}$ such that $t=$ $\left.\varphi \circ u_{\psi}(x, R)-\gamma_{1}\right\}$ and $Y=\left\{v \in \mathbb{R} \mid \exists N \in \mathcal{N},\left(x_{N}, R_{N}\right) \in(\mathcal{X} \times \mathcal{R})^{N}\right.$ such that $v=$ $\left.\sum_{i \in N} \varphi \circ u_{\psi}\left(x_{i}, R_{i}\right)-\sum_{i=2}^{|N|+1} \gamma_{i}\right\}$. Note that $T$ and $Y$ are intervals in $\mathbb{R}$ such that $0 \in T$, and $\mathbb{R}_{+}$is a subset of $Y$.

Let us define a relation $\succsim^{\star \star}$ on $T \times Y \times Y$ as follows. For all $(t, y, \hat{y}),\left(t^{\prime}, y^{\prime}, \hat{y}^{\prime}\right) \in$ $T \times Y \times Y,(t, y, \hat{y}) \succsim^{\star \star}\left(t^{\prime}, y^{\prime}, \hat{y}^{\prime}\right)$ if and only if $f \succsim g$ where $f, g \in \bar{F}$ are defined as follows:

- there exists $E \in \mathcal{E}$ with $p(E)=\pi$;

- there exist $N, N^{\prime}, M, M^{\prime} \in \mathcal{N}$ with $1 \notin N, 1 \notin N^{\prime}, 1 \notin M, 1 \notin M^{\prime}$, $N \cap M=\emptyset$ and $N^{\prime} \cap M^{\prime}=\emptyset$;

- there exist $\left(x_{1}, R_{1}\right),\left(x_{1}^{\prime}, R_{1}^{\prime}\right) \in \mathcal{X} \times \mathcal{R}$, such that $t=\varphi \circ u_{\psi}\left(x_{1}, R_{1}\right)-\gamma_{1}$ and $t^{\prime}=\varphi \circ u_{\psi}\left(x_{1}^{\prime}, R_{1}^{\prime}\right)-\gamma_{1}$

- there exist $\left(x_{N}, R_{N}\right) \in(\mathcal{X} \times \mathcal{R})^{N},\left(x_{N^{\prime}}^{\prime}, R_{N^{\prime}}^{\prime}\right) \in(\mathcal{X} \times \mathcal{R})^{N^{\prime}}$, such that $y=$ $\sum_{i \in N} \varphi \circ u_{\psi}\left(x_{i}, R_{i}\right)-\sum_{i=2}^{|N|+1} \gamma_{i}$ and $y^{\prime}=\sum_{i \in N^{\prime}} \varphi \circ u_{\psi}\left(x_{i}^{\prime}, R_{i}^{\prime}\right)-\sum_{i=2}^{\left|N^{\prime}\right|+1} \gamma_{i}$;

- there exist $\left(\hat{x}_{M}, \hat{R}_{M}\right) \in(\mathcal{X} \times \mathcal{R})^{\hat{M}},\left(\hat{x}_{M^{\prime}}^{\prime}, \hat{R}_{M^{\prime}}^{\prime}\right) \in(\mathcal{X} \times \mathcal{R})^{M^{\prime}}$, such that $\hat{y}=$ $\sum_{i \in M} \varphi \circ u_{\psi}\left(\hat{x}_{i}, \hat{R}_{i}\right)-\sum_{i=2}^{|M|+1} \gamma_{k}$ and $\hat{y}^{\prime}=\sum_{i \in M^{\prime}} \varphi \circ u_{\psi}\left(\hat{x}_{i}^{\prime}, \hat{R}_{i}^{\prime}\right)-\sum_{i=2}^{\left|M^{\prime}\right|+1} \gamma_{i} ;$ 
and $f(s)=\left(\left(x_{1}, R_{1}\right),\left(x_{n}, R_{N}\right)\right), g(s)=\left(\left(x_{1}^{\prime}, R_{1}^{\prime}\right),\left(x_{n}^{\prime}, R_{N^{\prime}}^{\prime}\right)\right)$ for all $s \in E$ while $f(s)=\left(\left(x_{1}, R_{1}\right),\left(\hat{x}_{n}, \hat{R}_{M}\right)\right)$ and $g(s)=\left(\left(x_{1}^{\prime}, R_{1}^{\prime}\right),\left(\hat{x}_{n}^{\prime}, \hat{R}_{M^{\prime}}^{\prime}\right)\right)$ for all $s \notin E$.

It can be verified that relation $\succsim^{\star \star}$ is a well-defined complete pre-order on $T \times Y \times Y$. By definition, for any $(t, y, \hat{y}),\left(t^{\prime}, y^{\prime}, \hat{y}^{\prime}\right) \in T \times Y \times Y$ :

$(t, y, \hat{y}) \succsim^{\star \star}\left(t^{\prime}, y^{\prime}, \hat{y}^{\prime}\right) \Longleftrightarrow \pi \Phi(t+y)+(1-\pi) \Phi(t+\hat{y}) \geq \pi \Phi\left(t^{\prime}+y^{\prime}\right)+(1-\pi) \Phi\left(t^{\prime}+\hat{y}^{\prime}\right)$

Also, by Independence of the sure, for any $t \in T,(y, \hat{y}),\left(y^{\prime}, \hat{y}^{\prime}\right) \in Y \times Y$, $(t, y, \hat{y}) \succsim^{\star \star}\left(t, y^{\prime}, \hat{y}^{\prime}\right) \Longleftrightarrow(0, y, \hat{y}) \succsim^{\star \star}\left(0, y^{\prime}, \hat{y}^{\prime}\right)$, and therefore

$$
\begin{array}{r}
\pi \Phi(t+y)+(1-\pi) \Phi(t+\hat{y}) \geq \pi \Phi\left(t+y^{\prime}\right)+(1-\pi) \Phi\left(t+\hat{y}^{\prime}\right) \\
\Phi(y)+(1-\pi) \Phi(\hat{y}) \geq \pi \Phi\left(y^{\prime}\right)+(1-\pi) \Phi\left(\hat{y}^{\prime}\right) .
\end{array}
$$

Denoting $\Psi_{t}: Y \rightarrow \mathbb{R}$ the function such that $\Psi_{t}(y)=\Phi(t+y)$, this implies that there exists a continuous and increasing function $\Theta_{t}: \mathbb{R} \rightarrow \mathbb{R}$ such that, for all $y, \hat{y} \in Y, \Theta_{t}(\pi \Phi(y)+(1-\pi) \Phi(\hat{y}))=\pi \Psi_{t}(y)+(1-\pi) \Psi_{t}(\hat{y})$. This is a Pexider functional equation, and it is known that in that case $\Theta_{t}$ and $\Psi_{t}$ must be affine (Radó and Baker, 1987). Hence there exist $\alpha_{t} \in \mathbb{R}_{++}$and $\beta_{t} \in \mathbb{R}$ such that $\Psi_{t}(y)=\alpha_{t} \Phi(y)+\beta_{t}$.

Define the functions $\alpha: T \rightarrow \mathbb{R}_{++}$and $\beta: T \rightarrow \mathbb{R}$ by $\alpha(t)=\alpha_{t}$ and $\beta(t)=\beta_{t}$ for all $t \in T$. By definition of function $\Psi_{t}$, we end up with the functional equation: $\Phi(t+y)=\alpha(t) \Phi(y)+\beta(t)$ for all $(t, w) \in T \times Y$. By Corollary 1 (pp. 150-151) in Aczél (1966), this equation implies that either $\Phi$ is affine or that it is an affine transformation of the function $y \rightarrow \alpha e^{\alpha y}$ for some $\alpha \neq 0$. Furthermore, we know that $\Phi$ is increasing.

Finally, we let $c_{n}=\sum_{i=1}^{n} \gamma_{i}$ (so that $c_{n} \in D_{\varphi}^{n}$ ) to obtain the desired formula. 


\section{A.3 Proof of Theorems 2 and 3}

\section{A.3.1 Proof of Theorem 2}

Assume that $\succsim$ is a fair social welfare ordering that satisfies Indifferent addition and Social expected utility. By Social expected utility there exists a function $V: \mathcal{A} \rightarrow \mathbb{R}$, which is unique up to increasing affine transformations, such that, for all $f, g \in \mathcal{F}$ :

$$
f \succsim g \Longleftrightarrow \mathbb{E}[V \circ f] \geq \mathbb{E}[V \circ g]
$$

By Proposition A.1, there exists a continuous and increasing set function $\psi$, a function $\Upsilon: \mathbb{N} \times \mathbb{R}_{+}$that is continuous and increasing in its second argument, and for each $n \in \mathbb{N}$ a continuous, symmetric, monotonic, and normalized function $W_{n}: \mathbb{R}_{+}^{n} \rightarrow \mathbb{R}$ such that, for all $f, g \in \overline{\mathcal{F}}$ :

$$
\begin{aligned}
f \succsim g \Longleftrightarrow & \mathbb{E}\left[\Upsilon\left(n(f), W_{n(f)}\left(\left(u_{\psi}\left(x_{i}(f), R_{i}(f)\right)\right)_{i \in N(f)}\right)\right)\right] \\
& \geq \mathbb{E}\left[\Upsilon\left(n(g), W_{n(g)}\left(\left(u_{\psi}\left(x_{i}(g), R_{i}(g)\right)\right)_{i \in N(g)}\right)\right)\right]
\end{aligned}
$$

Given that $V$ is unique up to an increasing affine transformation, it must be the case that there exist $\kappa \in \mathbb{R}_{+}$and $\gamma \in \mathbb{R}$ such that, for all $a \in \mathcal{A}$ :

$$
V(a)=\kappa \Upsilon\left(n(a), W_{n(a)}\left(\left(u_{\psi}\left(x_{i}, R_{i}\right)\right)_{i \in N(a)}\right)\right)+\gamma
$$

If in addition $\succsim$ satisfies Extended replication invariance, we know by Proposition A.2 that there exists a continuous, symmetric, normalized, replicationinvariant, and increasing function $\Gamma: \mathcal{Z} \rightarrow \mathbb{R}, \alpha \in \mathbb{R}$ and $\beta \in \mathbb{R}$ such that for all $n \in \mathbb{N}$ and $z \in \mathbb{R}_{+}^{n}$ :

$$
\Upsilon\left(n, W_{n}(z)\right)=\left\{\begin{array}{l}
n^{\alpha}(\Gamma(z)-\beta) \text { if } \alpha \neq 0 \\
\Gamma(z)+\beta \ln (n) \text { if } \alpha=0
\end{array}\right.
$$

On the other hand, if in addition $\succsim$ satisfies Independence of the sure, we know by Proposition A.3 that a constant $\alpha \in \mathbb{R}$, a function $\varphi: \mathbb{R}_{+} \rightarrow \mathbb{R}$, and for 
each $n \in \mathbb{N}$ a constant $c_{n} \in D_{\varphi}^{n}$ such that, for all $z \in \mathbb{R}_{+}^{n}$ :

$$
\Upsilon\left(n, W_{n}(z)\right)=\left\{\begin{array}{l}
\sum_{i=1}^{n} \varphi\left(z_{i}\right)-c_{n} \text { if } \alpha=0 \\
\frac{1}{\alpha} e^{\alpha \sum_{i=1}^{n} \varphi\left(z_{i}\right)-c_{n}} \text { if } \alpha \neq 0
\end{array}\right.
$$

Renaming $\Upsilon$ as $V, W_{n}$ and $\Gamma$ as $W$, one obtains the formulation of the theorem.

\section{A.3.2 Proof of Theorem 3}

Assume that $\succsim$ is a fair social welfare ordering that satisfies Indifferent addition, Pareto and and Restricted social expected utility. By Proposition A.1, there exists a continuous and increasing set function $\psi$, a function $V: \mathbb{N} \times \mathbb{R}_{+}$that is continuous and increasing in its second argument, and for each $n \in \mathbb{N}$ a continuous, symmetric, monotonic, and normalized function $W_{n}: \mathbb{R}_{+}^{n} \rightarrow \mathbb{R}$ such that, for all $f, g \in \overline{\mathcal{F}}$ :

$$
\begin{aligned}
f \succsim g \Longleftrightarrow & \mathbb{E}\left[V\left(n(f), W_{n(f)}\left(\left(u_{\psi}\left(x_{i}(f), R_{i}(f)\right)\right)_{i \in N(f)}\right)\right)\right] \\
& \geq \mathbb{E}\left[V\left(n(g), W_{n(g)}\left(\left(u_{\psi}\left(x_{i}(g), R_{i}(g)\right)\right)_{i \in N(g)}\right)\right)\right]
\end{aligned}
$$

If in addition $\succsim$ satisfies Extended replication invariance, we know by Proposition A.2 that there exists a continuous, symmetric, replication-invariant, normalized and increasing function $\Gamma: \mathcal{Z} \rightarrow \mathbb{R}, \alpha \in \mathbb{R}$ and $\beta \in \mathbb{R}$ such that for all $n \in \mathbb{N}$ and $z \in \mathbb{R}_{+}^{n}$ :

$$
V\left(n, W_{n}(z)\right)=\left\{\begin{array}{l}
n^{\alpha}(\Gamma(z)-\beta) \text { if } \alpha \neq 0 \\
\Gamma(z)+\beta \ln (n) \text { if } \alpha=0
\end{array}\right.
$$

On the other hand, if in addition $\succsim$ satisfies Independence of the sure, we know by Proposition A.3 that a constant $\alpha \in \mathbb{R}$, a function $\varphi: \mathbb{R}_{+} \rightarrow \mathbb{R}$, and for each $n \in \mathbb{N}$ a constant $c_{n} \in D_{\varphi}^{n}$ such that, for all $z \in \mathbb{R}_{+}^{n}$ :

$$
V\left(n, W_{n}(z)\right)=\left\{\begin{array}{l}
\sum_{i=1}^{n} \varphi\left(z_{i}\right)-c_{n} \text { if } \alpha=0 \\
\frac{1}{\alpha} e^{\alpha \sum_{i=1}^{n} \varphi\left(z_{i}\right)-c_{n}} \text { if } \alpha \neq 0
\end{array}\right.
$$


Now consider any $f, g \in \mathcal{F}$. Construct $\bar{f}, \bar{g} \in \overline{\mathcal{F}}$ as follows: for any $s \in \mathcal{S}$,

- $N(f(s))=N(\bar{f}(s))$ and $N(g(s))=N(\bar{g}(s))$;

- $R_{i}(\bar{f}(s))=R_{i}(f(s))$ and $x_{i}(\bar{f}(s))=c e_{i, R_{i}}(f)$ for all $i \in N(f(s))$;

- and $R_{i}(\bar{g}(s))=R_{i}(g(s))$ and $x_{i}(\bar{g}(s))=c e_{i, R_{i}}(g)$ for all $i \in N(g(s))$.

So in every state of the world, the people who exist are the same in $f$ and $\bar{f}$. Each of these people has the same preferences in $f$ and $\bar{f}$, but in $\bar{f}$ they receive the certainty-equivalent. And a similar comparison holds between $g$ and $\bar{g}$. By Pareto (and the definition of a certainty-equivalent), $f \sim \bar{f}$ and $g \sim \bar{g}$. Hence, $f \succsim g \Longleftrightarrow \bar{f} \succsim \bar{g}$. But $\bar{f}, \bar{g} \in \overline{\mathcal{F}}$ so that:

$$
\begin{aligned}
\bar{f} \succsim \bar{g} & \Longleftrightarrow \mathbb{E}\left[V\left(n(\bar{f}), W_{n(\bar{f})}\left(\left(u_{\psi}\left(x_{i}(\bar{f}), R_{i}(\bar{f})\right)_{i \in N(\bar{f})}\right)\right)\right]\right. \\
& \geq \mathbb{E}\left[V\left(n(\bar{g}), W_{n(\bar{g})}\left(\left(u_{\psi}\left(x_{i}(\bar{g}), R_{i}(\bar{g})\right)\right)_{i \in N(\bar{g})}\right)\right)\right] \\
\Longleftrightarrow & \sum_{\left(N, R_{N}\right)} p_{N, R_{N}}(f) \times V\left(|N|, W_{|N|}\left(\left(u_{\psi}\left(c e_{i, R_{i}}(f), R_{i}\right)\right)_{i \in N}\right)\right) \geq \\
& \sum_{\left(N, R_{N}\right)} p_{N, R_{N}}(g) \times V\left(|N|, W_{|N|}\left(\left(u_{\psi}\left(c e_{i, R_{i}}(g), R_{i}\right)\right)_{i \in N}\right)\right) .
\end{aligned}
$$

\section{A.4 Proof of Theorem 4}

We start with an extension of the welfarism theorem to cases where the social welfare ordering satisfies Pareto for individual allocation and Social expected utility.

Proposition A.4 If a social welfare ordering $\succsim$ on $\mathcal{F}$ satisfies Pareto for individual risk, Social expected utility, Betterness dominance, and Anonymity, then there exists for each $n \in \mathbb{N}$, a continuous, symmetric, and monotonic function $\bar{W}_{n}: \mathbb{R}^{n} \rightarrow \mathbb{R}$ such that for all $f, g \in \mathcal{F}$ :

$f \succsim g \Longleftrightarrow \mathbb{E}\left[\bar{W}_{n(f)}\left(\left(\tilde{v}_{R_{i}(f)}\left(x_{i}(f)\right)\right)_{i \in N(f)}\right)\right] \geq \mathbb{E}\left[\bar{W}_{n(g)}\left(\left(\tilde{v}_{R_{i}(g)}\left(x_{i}(g)\right)\right)_{i \in N(g)}\right)\right]$.

where for each $R \in \mathcal{R}, \tilde{v}_{R}$ is a VNM utility function. 
Proof. Consider any $i \in \mathbb{N}, R_{i} \in \mathcal{R}$, and any $f, g \in \mathcal{F}$ such that $N(f(s))=$ $N(g(s))=\{i\}$ and $R_{i}(f(s))=R_{i}(g(s))=R_{i}$ for all $s \in \mathcal{S}$. By Pareto for equal allocation and social expected utility:

$$
f \succ g \Longleftrightarrow \mathbb{E}\left[v_{R_{i}}\left(x_{i}(f)\right)\right]>\mathbb{E}\left[v_{R_{i}}\left(x_{i}(g)\right)\right] \Longleftrightarrow \mathbb{E}[V \circ f]>\mathbb{E}[V \circ g]
$$

By unicity of the VNM function up to an increasing affine transformation, there must exist $\alpha_{R_{i}}^{i} \in \mathbb{R}_{++}$and $\beta_{R_{i}}^{i} \in \mathbb{R}$ such that $V(f)=\alpha_{R_{i}}^{i} v_{R_{i}}\left(x_{i}\right)+\beta_{R_{i}}^{i}$ for all $x_{i} \in \mathcal{X}$. By Anonymity, $V(f)$ does not depend on $i$ 's identity, implying that $\alpha_{R_{i}}^{i}=\alpha_{R_{i}}$ and $\beta_{R_{i}}^{i}=\beta_{R_{i}}$ for some $\alpha_{R_{i}}, \beta_{R_{i}}$.

Denote $\tilde{v}_{R}=\alpha_{R} v_{R}+\beta_{R}$. By the previous point, for all $i, j$, for all $\left(x_{i}, R_{i}\right),\left(x_{j}, R_{j}\right) \in$ $\mathcal{X} \times \mathcal{R},\left(x_{i}, R_{i}\right) \succ\left(x_{j}, R_{j}\right)$ if and only if $\tilde{v}_{R_{i}}\left(x_{i}\right)>\tilde{v}_{R_{j}}\left(x_{j}\right)$. By Betterness dominance, Anonymity, and continuity of the social welfare ordering $\succsim$, we have that for all $N, M \in \mathcal{N}$ with $|N|=|M|=n$, all $R_{N} \in \mathcal{R}^{N}, R_{M}^{\prime} \in \mathcal{R}^{M}, x_{N} \in \mathcal{X}^{N}$ and $x_{M}^{\prime} \in \mathcal{X}^{M}$, if there exist a bijection $\sigma: N \rightarrow M$ such that for all $i \in N$ $\tilde{v}_{R_{i}}\left(x_{i}\right)=\tilde{v}_{R_{\sigma(i)}^{\prime}}\left(x_{\sigma(i)}^{\prime}\right)$ then $\left(x_{N}, R_{N}\right) \sim\left(x_{M}^{\prime}, x_{M}^{\prime}\right)$.

Let $R^{\star}$ be a preference ordering for which there exists $\omega^{\star} \in \mathcal{X} \backslash\{0\}$ such that $\tilde{v}_{R^{\star}}(x)=a \ln \left(e_{\omega^{\star}}\left(x, R^{\star}\right)\right)+b$, with $a \in \mathbb{R}_{++}$and $b \in \mathbb{R}$, for all $x \in \mathcal{X} .{ }^{12}$ Consider any $n \in \mathbb{N}$ and let $N_{n}=\{1, \cdots, n\}$ and $\hat{R}_{n}=\left(R^{\star}, \cdots, R^{\star}\right)$. Let $\succsim_{n}^{\star \star}$ be a binary relation defined on $\mathbb{R}^{n}$ as follows: for all $z, z^{\prime} \in \mathbb{R}^{n} z \succsim_{n}^{\star \star} z^{\prime}$ if and only if there is $x_{N_{n}}, x_{N_{n}}^{\prime} \in \mathcal{X}^{N_{n}}$ such that $z_{i}=\tilde{v}_{R^{\star}}\left(x_{i}\right)$ and $z_{i}^{\prime}=\tilde{v}_{R^{\star}}\left(x_{i}^{\prime}\right)$ all $i \in N_{n}$, and $\left(x_{N_{n}}, \hat{R}_{n}\right) \succsim\left(x_{N_{n}}^{\prime}, \hat{R}_{n}\right)$. This relation is well defined: if $\hat{x}_{N_{n}}, \hat{x}_{N_{n}}^{\prime} \in \mathcal{X}^{N_{n}}$ are such that $z_{i}=\tilde{v}_{R^{\star}}\left(\hat{x}_{i}\right)$ and $z_{i}^{\prime}=\tilde{v}_{R^{\star}}\left(\hat{x}_{i}^{\prime}\right)$ all $i \in N_{n}$, then $\left(x_{N_{n}}, \hat{R}_{n}\right) \sim\left(\hat{x}_{N_{n}}, \hat{R}_{n}\right)$ and $\left(x_{N_{n}}^{\prime}, \hat{R}_{n}\right) \sim\left(\hat{x}_{N_{n}}^{\prime}, \hat{R}_{n}\right)$. By definition, relation $\succsim_{n}^{\star \star}$ is transitive, complete and continuous. Hence there exists a continuous function $W_{n}: \mathbb{R}^{n} \rightarrow \mathbb{R}$ such that, for all $z, z^{\prime} \in \mathbb{R}^{n}$ :

$$
z \succsim^{\star} z^{\prime} \Longleftrightarrow W_{n}(z) \geq W_{n}\left(z^{\prime}\right)
$$

Betterness dominance and Anonymity imply that $W_{n}$ is monotonic and symmet-

\footnotetext{
${ }^{12}$ Recall that $e_{\omega}(x, R)$ is the equivalent level of the Pazner-Schmeidler equivalence approach: see Section 2.2.
} 
ric. And by definition, for all $x_{N_{n}}, x_{N_{n}}^{\prime} \in \mathcal{X}^{N_{n}}$ :

$$
\left(x_{N_{n}}, \hat{R}_{n}\right) \succsim\left(x_{N_{n}}^{\prime}, \hat{R}_{n}\right) \Longleftrightarrow W_{n}\left(\left(\tilde{v}_{R^{\star}}\left(x_{i}\right)\right)_{i \in N_{n}}\right) \geq W_{n}\left(\left(\tilde{v}_{R^{\star}}\left(x_{i}^{\prime}\right)\right)_{i \in N_{n}}\right) .
$$

Consider any $N, M \in \mathcal{N}$ with $|N|=|M|=n$, as well as $R_{N} \in \mathcal{R}^{N}, R_{M}^{\prime} \in$ $\mathcal{R}^{M}, x_{N} \in \mathcal{X}^{N}$ and $x_{M}^{\prime} \in \mathcal{X}^{M}$. Let $\sigma: N \rightarrow N_{n}$ and $\hat{\sigma}: M \rightarrow N_{n}$ be two bijections. Let $\hat{x}_{N_{n}}, \tilde{x}_{N_{n}} \in \mathcal{X}^{N_{n}}$ be defined by $\tilde{v}_{R^{\star}}\left(\hat{x}_{\sigma(i)}\right)=\tilde{v}_{R_{i}}\left(x_{i}\right)$ for all $i \in N$ and $\tilde{v}_{R^{\star}}\left(\tilde{x}_{\sigma(j)}\right)=\tilde{v}_{R_{j}}\left(x_{j}^{\prime}\right)$ for all $j \in M .{ }^{13}$ By the above results, we know that for each $i \in N,\left(x_{i}, R_{i}\right) \sim\left(\hat{x}_{\sigma(i)}, R^{\star}\right)$, and for each $j \in M,\left(x_{j}^{\prime}, R_{j}^{\prime}\right) \sim\left(\tilde{x}_{\hat{\sigma}(j)}, R^{\star}\right)$. Therefore, $\left(x_{N}, R_{N}\right) \sim\left(\hat{x}_{N_{n}}, \hat{R}_{n}\right)$ and $\left(x_{M}^{\prime}, R_{M}^{\prime}\right) \sim\left(\tilde{x}_{N_{n}}, \hat{R}_{n}\right)$.

Gathering all our results, we obtain the following equivalence:

$$
\begin{aligned}
\left(x_{N}, R_{N}\right) \succsim\left(x_{M}^{\prime}, R_{M}^{\prime}\right) & \Longleftrightarrow\left(\hat{x}_{N_{n}}, \hat{R}_{n}\right) \succsim\left(\tilde{x}_{N_{n}}, \hat{R}_{n}\right) \\
& \Longleftrightarrow W_{n}\left(\left(\tilde{v}_{R^{\star}}\left(\hat{x}_{i}\right)\right)_{i \in N_{n}}\right) \geq W_{n}\left(\left(\tilde{v}_{R^{\star}}\left(\tilde{x}_{i}\right)\right)_{i \in N_{n}}\right) \\
& \Longleftrightarrow W_{n}\left(\left(\tilde{v}_{R_{i}}\left(x_{i}\right)\right)_{i \in N}\right) \geq W_{n}\left(\left(\tilde{v}_{R_{j}}\left(x_{j}\right)\right)_{j \in M}\right),
\end{aligned}
$$

where the last equivalence is due to the symmetry of $W_{n}$.

By Social expected utility, we also know that for all $n \in \mathbb{N}$, all $N, M \in \mathcal{N}$ with $|N|=|M|=n$, all $R_{N} \in \mathcal{R}^{N}, R_{M}^{\prime} \in \mathcal{R}^{M}, x_{N} \in \mathcal{X}^{N}$ and $x_{M}^{\prime} \in \mathcal{X}^{M}$ :

$$
\left(x_{N}, R_{N}\right) \succsim\left(x_{M}^{\prime}, R_{M}^{\prime}\right) \Longleftrightarrow V\left(\left(\left(x_{i}, R_{i}\right)\right)_{i \in N}\right) \geq V\left(\left(\left(x_{j}, R_{j}\right)\right)_{j \in M}\right)
$$

So there must exist a continuous and increasing function $\Psi_{n}$ such that, for all $N$

${ }^{13}$ Note that these allocations are well-defined. For instance, if $\tilde{v}_{R_{i}}\left(x_{i}\right)=z \in \mathbb{R}$, we can define the allocation $\hat{x}_{\sigma(i)}=\lambda \omega^{\star}$, with $\lambda=\exp (z / a-b / a)$, so that, by definition of $R^{\star}$,

$$
\tilde{v} R \star(\hat{x} \sigma(i))=a \ln (e \omega \star(x, R \star))+b=a \ln (\exp (z / a-b / a))+b=z=\tilde{v} R i(x i) .
$$

We can proceed in the same way to define $\tilde{x}_{N_{n}}$. 
with $|N|=n$, any $\left(x_{N}, R_{N}\right) \in \mathcal{X}^{N} \times \mathcal{R}^{N}$ :

$$
V\left(\left(\left(x_{i}, R_{i}\right)\right)_{i \in N}\right)=\Psi_{n}\left(W_{n}\left(\left(\tilde{v}_{R_{i}}\left(x_{i}\right)\right)_{i \in N}\right)\right)=\bar{W}_{n}\left(\left(\tilde{v}_{R_{i}}\left(x_{i}\right)\right)_{i \in N}\right)
$$

where $\bar{W}_{n}=\Psi_{n} \circ W_{n}$.

Assume that $\succsim$ satisfies Betterness dominance, Anonymity, Indifferent addition, Pareto for equal risk and Social expected utility. Pareto for equal risk implies Pareto for individual risk, so that Proposition A.4 holds. Now, consider any $n \in \mathbb{N}$ and let $f, g \in \mathcal{F}$ be two prospects such that, for each $s \in \mathcal{S}, N(s)=N_{n}$, $R_{N(s)}(f(s))=R_{N(s)}(g(s))=\hat{R}_{n}=\left(R^{\star}, \cdots, R^{\star}\right)$, and $x_{i}(f(s))=x_{j}(f(s))$ and $x_{i}(g(s))=x_{j}(g(s))$ for all $i, j \in N_{n}$ : these are prospects where only people with preferences $R^{\star}$ exist and they all have the same allocation ex post. Denote by $\tilde{\phi}_{n}$ the function such that $\tilde{\phi}_{n}(z)=\bar{W}_{n}(\underbrace{z, \cdots, z}_{n \text { times }})$. By Proposition A.4:

$$
f \succsim g \Longleftrightarrow \mathbb{E}\left[\tilde{\phi}_{n}\left(\tilde{v}_{R^{\star}}\left(x_{1}(f)\right)\right)\right] \geq \mathbb{E}\left[\tilde{\phi}_{n}\left(\tilde{v}_{R^{\star}}\left(x_{1}(g)\right)\right)\right]
$$

But $f$ and $g$ induce equal risk, so that by Pareto for equal risk:

$$
f \succsim g \Longleftrightarrow \mathbb{E}\left[\tilde{v}_{R^{\star}}\left(x_{1}(f)\right)\right] \geq \mathbb{E}\left[\tilde{v}_{R^{\star}}\left(x_{1}(g)\right)\right] .
$$

Given that $\tilde{v}_{R^{\star}}(\mathcal{X})=\mathbb{R}$, it clearly needs to be the case that for all $z \in \mathbb{R} \tilde{\phi}_{n}(z)=$ $\alpha_{n} z+\beta_{n}$, with $\alpha_{n} \in \mathbb{R}_{++}$and $\beta_{n} \in \mathbb{R}$. Therefore, for each $n \in \mathbb{N}$ there exist a continuous, symmetric, monotonic, and normalized function $W_{n}: \mathbb{R}_{+}^{n} \rightarrow \mathbb{R}_{+}$such that $\bar{W}_{n}=\alpha_{n} W_{n}+\beta_{n}$, where $\bar{W}_{n}$ is the function in Proposition A.4. We thus obtain the first part of Theorem 4 .

Given the form of social welfare orderings satisfying Pareto for individual risk, Social expected utility, Betterness dominance, and Anonymity (Proposition A.4), and the fact that $\succsim$ also satisfies Indifferent addition, we can repeat the reasoning in Propositions A.2 and A.3 to show that:

1. If $\succsim$ also satisfies Extended replication invariance, there exist a continuous, 
symmetric, monotonic, normalized and replication-invariant function $\Gamma$ : $\mathcal{Z} \rightarrow \mathbb{R}, \alpha \in \mathbb{R}$ and $\beta \in \mathbb{R}$ such that, for all $z \in \mathcal{Z}$ :

$$
\alpha_{n(z)} W_{n(z)}(z)+\beta_{n(z)}= \begin{cases}n(z)^{\alpha}(\Gamma(z)-\beta) & \text { if } \alpha \neq 0 \\ \Gamma(z)+\beta \ln (n(z)) & \text { if } \alpha=0\end{cases}
$$

2. If $\succsim$ also satisfies Independence of the sure, then there must exist a constant $\alpha \in \mathbb{R}$, a function $\varphi: \mathbb{R} \rightarrow \mathbb{R}$, and for each $n \in \mathbb{N}$ a constant $c_{n} \in D_{\varphi}^{n}$ such that, for all $z \in \mathcal{Z}$ :

$$
\alpha_{n(z)} W_{n(z)}(z)+\beta_{n(z)}=\left\{\begin{array}{cl}
\frac{1}{\alpha} e^{\alpha \sum_{i \in N(z)} \varphi\left(z_{i}\right)-c_{n(z)}} & \text { if } \alpha \neq 0 \\
\sum_{i \in N(z)} \varphi\left(z_{i}\right)-c_{n(z)} & \text { if } \alpha=0
\end{array}\right.
$$

But we should have $\alpha_{n} W_{n}(\underbrace{t, \cdots, t}_{n \text { times }})+\beta_{n}=\alpha_{n} t+\beta_{n}$ for all $t \in \mathbb{R}$ and $n \in \mathbb{N}$.

(a) For the formula with $\alpha \neq 0$, we would get $\frac{\left(e^{\alpha \varphi(t)}\right)^{n}}{\alpha e^{\alpha c_{n}}}$ which cannot always be equal to $\alpha_{n} t+\beta_{n}$ when $t$ varies. So the case $\alpha \neq 0$ cannot hold.

(b) For the formula with $\alpha=0$, we would get $n \varphi(t)-c_{n}$ which can always be equal to $\alpha_{n} t+\beta_{n}$ when $t$ varies only if $\varphi$ is an increasing affine function. 